\title{
Selection of stimulus parameters for enhancing slow wave sleep events with a Neural-field theory thalamocortical computational model
}

Felipe A. Torres ${ }^{1,3}$, Patricio Orio ${ }^{2,3, *}$, María-José Escobar ${ }^{1, *}$

1 Department of Electronic Engineering, Universidad Técnica Federico Santa María, Valparaíso, Chile

2 Centro Interdisciplinario de Neurociencia de Valparaíso, Universidad de Valparaíso, Valparaíso, Chile

3 Advanced Center for Electrical and Electronic Engineering (AC3E), Valparaíso, Chile

All authors contributed equally to this work.

* patricio.orio@uv.cl, mariajose.escobar@usm.cl 


\begin{abstract}
Slow-wave sleep cortical brain activity, conformed by slow-oscillations and sleep spindles, plays a key role in memory consolidation. The increase of the power of the slow-wave events, obtained by auditory sensory stimulation, positively correlates to memory consolidation performance. However, little is known about the experimental protocol maximizing this effect, which could be induced by the power of slow-oscillation, the number of sleep spindles, or the timing of both events' co-occurrence. Using a mean-field model of thalamocortical activity, we studied the effect of several stimulation protocols, varying the pulse shape, duration, amplitude, and frequency, as well as a target-phase using a closed-loop approach. We evaluated the effect of these parameters on slow-oscillations ( $\mathrm{SO}$ ) and sleep-spindles (SP), considering: (i) the power at the frequency bands of interest, (ii) the number of SO and SP, (iii) co-occurrences between SO and SP, and (iv) synchronization of SP with the up-peak of the SO. The first three targets are maximized using a decreasing ramp pulse with a pulse duration of $50 \mathrm{~ms}$. Also, we observed a reduction in the number of SO when increasing the stimulus energy by rising its amplitude. To assess the target-phase parameter, we applied closed-loop stimulation at $0^{\circ}, 45^{\circ}$, and $90^{\circ}$ of the phase of the narrow-band filtered ongoing activity, at $0.85 \mathrm{~Hz}$ as central frequency. The $0^{\underline{0}}$ stimulation produces better results in the power and number of SO and SP than the rhythmic or aleatory stimulation. On the other hand, stimulating at $45^{\mathrm{O}}$ or $90^{\mathrm{O}}$ change the timing distribution of spindles centers but with fewer co-occurrences than rhythmic and $0^{\underline{0}}$ phase. Finally, we propose the application of closed-loop stimulation at the rising zero-cross point using pulses with a decreasing ramp shape and $50 \mathrm{~ms}$ of duration for future experimental work.
\end{abstract}

\title{
Author summary
}

During the non-REM (NREM) phase of sleep, events that are known as slow oscillations (SO) and spindles (SP) can be detected by EEG. These events have been associated with the consolidation of declarative memories and learning. Thus, there is an ongoing interest in promoting them during sleep by non-invasive manipulations such as sensory stimulation. In this paper, we used a computational model of brain activity that generates SO and SP, to investigate which type of sensory stimulus -shape, amplitude, duration, periodicity- would be optimal for increasing the events' frequency and their co-occurrence. We found that a decreasing ramp of $50 \mathrm{~ms}$ duration is the most effective. The effectiveness increases when the stimulus pulse is delivered in a closed-loop configuration triggering the pulse at a target phase of the ongoing SO activity. A desirable secondary effect is to promote SPs at the rising phase of the SO oscillation.

\section{Introduction}

Humans spend about one-third time of their life sleeping. This behavior has paramount importance for the process of learning, as it contributes to the consolidation of memories 1, 2. During the non-rapid eye movement (NREM) phase of sleep, the brain's electrical activity is characterized by the occurrence of events recognizable on the cortical activity measured by electroencephalogram (EEG) registers. These events are the slow oscillations ( $\mathrm{SO}$ ), single high-amplitude cortical oscillations lasting 0.8 to 2 seconds 3 ; and the sleep spindles (SP), thalamocortical oscillation bursts lasting 0.5 to 2 seconds in the frequency band 9-16 $\mathrm{Hz}$ [4. NREM is also recognizable by events on the hippocampus activity, the sharp wave-ripples, oscillations bursts in the frequency range 100-250 $\mathrm{Hz}$ with $50-100 \mathrm{~ms}$ duration that can be measured with invasive intracranial electrodes [5]. 
Memory consolidation is a complex problem where different mechanisms play a role. The temporal coincidence of sharp wave-ripples, the sleep spindles, and the slow oscillations promote the transformation of short-term memories stored in hippocampus connections to long-term storage memories by incrementing the synaptic strength of cortical neural networks. This perspective is known as the Standard Consolidation Theory [6], and only occurs during the deepest NREM sleep stage [5,7]. On the other hand, REM sleep theta-gamma oscillations coupling, and the alternation between REM and NREM stages also impact memory consolidation [8].

Regarding slow wave sleep (SWS), NREM stage of slow frequency activity with SOs and SPs' presence, the literature points that the number of spindles appearances locked to the UP phase of slow oscillations could play a more significant role in cortical memory formation 9 11. This has led to a standing interest in improving the consolidation process -and thus long-term memory- by non-invasive sensory stimulation or direct electromagnetic stimulation while the brain is in SWS 12, 13 .

The physiological goal of stimulation during SWS is to increase the power and the occurrences of both events (SO and SPs), including their precise timing coincidence. An increase of sharp-wave ripples is also desirable [14, but there is no way to measure hippocampus activity with non-invasive methods. Further, stimulation for memory consolidation improvement should not disturb sleep cycles [2]. Stimulating during SWS also takes advantage of less awareness on this sleep stage to external stimuli.

There is evidence supporting that sensory manipulation of SO leads to memory improvement [3] or disruption [15], with significant effects in enhancing the declarative memory and no impact on procedural memory 15, 16. Moreover, the number of SPs during SWS, and their power, is also related with best results in word-pairs memory tests [2]. However, the knowledge and optimization of stimulation protocols have been based on correlation analysis rather than causal evidence [17].

Even if auditory closed-loop stimulation has been used to improve the memory consolidation process, there is no evidence of tuning the stimulation protocol to maximize this effect. Specifically, sensory stimulation looks to maximize the correct timing between the evoked SP and SO events associated with the memory consolidation process. The manipulation of SP in auditory closed-loop [18, and the use of memory cues (sounds related to the learning episode), show better results when the external stimulus is applied at a specific phase of the $\mathrm{SO}[19$.

An alternative approach to improving the stimulation paradigm that attempts to increase SP and SO events during SWS is the use of mathematical models of brain activity 20 21. In this way, mechanistic insights about the generation of SP and SO activity can guide the search for a better stimulation that maximizes its efficacy. In this work, we present a systematic study of possible stimulation protocols and the more efficient features of the stimuli in promoting these SWS events' co-occurrence.

Specifically, in this work we use a model based on the Neural Field Theory (NFT) 22, 23] with corticothalamic loops between two cortical neural populations: excitatory or pyramidal $(e)$ and inhibitory or interneurons $(i)$, and two thalamic populations: reticular nucleus $(r)$ and relay nuclei $(s)$. This model is appropriate for the proposed exploration as it simulates the cerebral structures that originate SO and SP, namely the cortico-thalamic loops. Moreover, this model has been previously tuned to reproduce the electrical brain activity in different states: from arousal to slow-wave sleep and including the different stages of NREM sleep 24,25]. In this NFT model, sensory stimulation can be introduced as spatial-localized perturbations on the noisy input neural population, obtaining an EEG-like signal as the model's output. From this signal, we can assess the efficacy of the stimulation paradigms and implement a closed-loop feedback mechanism that can fine-tune the stimuli delivery timing.

With the purpose of simulating a single-modality sensory stimulation, we searched 
for a one-channel impulse pattern that alters the dynamics of the entire cortex. We assessed the efficacy of different pulse shapes, delivered with different frequency, intensity, and periodicity, including a closed-loop algorithm that intended to phase-lock the stimuli with the SO. The stimulation goals were:

1. To increase the power at both frequency bands of interest (SO and SP), measured by the scalogram (wavelet spectrogram).

2. To increase the quantity of SO and SP events and detecting their occurrence on the time series.

3. To rise the co-occurrences between SO and SP events, over the level of co-occurrences obtained by chance.

4. To increment the occurrence of SP on the up-peak of the SO, measured by the delay time between the down-peak of the slow oscillation and the center of the coincident sleep spindle.

Our results propose a sensory stimulation protocol maximizing the $\mathrm{SP}$ and SO co-occurrence associated with the memory consolidation process. Specifically, the SP and SO events' best co-occurrence is obtained when $50 \mathrm{~ms}$ decreasing ramp pulses were delivered at the zero phases (sine reference) of the ongoing activity filtered at a specific central frequency on the SO band. Nevertheless, the behavioral effect of this stimulation protocol on memory performance should be evaluated with clinical experiments.

\section{Results}

\section{Selection of model parameters for SWS stage}

In EEG recordings, the classification as NREM sleep comes from the presence of SWS events on epochs of 30 seconds. The prominence of SO indicates the N3 stage, and the occurrence of SPs indicates the N2 stage 26 . Furthermore, the evidence suggests that $\mathrm{SP}$ nested in the up phase of the SO may play a role in memory consolidation [10]. A SWS model, including SOs and SPs, presents advantages to characterize the conditions promoting their co-occurrence. Previous works using the eirs-NFT model were specific for the N2 stage (only SP) [24], or for the N3 stage only (without SP) [25]. Then, our first goal will be to tune the model parameters into a regime that exhibits both events.

In the eirs-NFT model, the sleep (and awake) stages can be represented as points in a XYZ space of model parameters, related to physiological connections. Fig 1 A shows this space where the $\mathrm{X}$-axis represents the strength of the intra-cortical connections, higher as sleep deepens. The Y-axis represents the internal thalamocortical connections that have a negative value during sleep. The more pronounced variations between sleep stages are in the Z-axis, representing the intra-thalamic connections [25]. In terms of the loop gains, the axes are defined as:

$$
\begin{aligned}
X & =\frac{G_{e e}}{1-G_{e i}} \\
Y & =\frac{G_{e s} G_{s e}+G_{e s} G_{r e} G_{s r}}{\left(1-G_{s r} G_{r s}\right)\left(1-G_{e i}\right)} \\
Z & =-G_{s r} G_{r s} \frac{\alpha \beta}{(\alpha+\beta)^{2}}
\end{aligned}
$$

where the gain-loops $G_{a b}$ are the same of Eq (4) and they are dependent of the connection strengths and the steady-state input $\phi_{n}^{(0)}$ (see Eq (5) and Eq (7) in Methods). Then the position in the XYZ space depends on the model parameters and $\phi_{n}^{(0)}$. 
Furthermore, Fig 1A shows excerpts of the cortical excitatory population activity for N2 and N3 stages, and we traced a trajectory between these two stages using linear interpolation on the connection strengths. Other examples of activity trace are shown within the linear interpolation and next to variations of the steady-state input value.

Dominant spindle activity is appreciable using the N2-stage parameters indicated in 24]. The spindle activity decreases as the simulation point slides along the interpolated trajectory towards the N3 stage, which shows slow-wave sleep dynamics but not noticeable spindle activity.

We are interested in an intermediate state between N2 and N3. For this, we concentrated on having energy both in N2 and N3 frequency bands. We obtained this condition in the second half of the linear interpolation between N2 and N3. Within this segment, we arbitrarily selected the point at 2/3 on the N2 to N3 trajectory. Fig $1 \mathrm{~B}$ shows the linearized model spectrums of N2 and N3 stages and three extra points in the interpolation trajectory, including the $2 / 3$ position in the blue-line.

The increase of the steady-state input value $\left(\phi_{n}^{(0)}\right)$ raises the model response in the Z-axis (Fig 1A), indicating lightness of NREM sleep [25,27]. Besides, it also increases the power and frequency of the SP-band peak (Fig $1 \mathrm{C})$, then a higher steady-state input value facilitates $\mathrm{SP}$ occurrence.

We selected $\phi_{n}^{(0)}=1$ as steady-state input in order to have similar peak amplitudes for frequency band lobes of both SWS events. The black line in Fig $1 \mathrm{C}$ shows the corresponding spectrum of the chosen simulation parameters. The peak frequency is close to the lower SP frequency with the selected parameters for the connections gains and the steady-state input. Table 1 presents the used parameters in our simulations of the eirs-NFT model.

Table 1. Parameters selected for SWS with spindles

\begin{tabular}{c|c|c} 
Symbol & Value & Unit \\
\hline $\mathrm{Q}$ & 340 & $s^{-1}$ \\
$\sigma_{\rho}$ & 0.0038 & $V$ \\
$\theta$ & 0.01292 & $V$ \\
$r_{e}$ & 0.086 & $m$ \\
$\gamma$ & 116 & $s^{-1}$ \\
$k_{0}$ & 10 & $m$ \\
$\alpha$ & 45 & $s^{-1}$ \\
$\beta$ & 186 & $s^{-1}$ \\
$t_{0}$ & 0.085 & $s$ \\
$\nu_{e e}$ & 5.54 & $m V s^{-1}$ \\
$\nu_{e i}$ & -5.65 & $m V s^{-1}$ \\
$\nu_{e s}$ & 1.53 & $m V s^{-1}$ \\
$\nu_{r e}$ & 0.286 & $m V s^{-1}$ \\
$\nu_{r s}$ & 1.12 & $m V s^{-1}$ \\
$\nu_{s e}$ & 2.69 & $m V s^{-1}$ \\
$\nu_{s r}$ & -1.73 & $m V s^{-1}$ \\
$\nu_{s n}$ & 9.22 & $m V s^{-1}$ \\
$\phi_{n}=\phi_{n}^{(0)}$ & 1 & $s^{-1}$ \\
$s t d\left(\phi_{n}\right)$ & 3.11 & $s^{-1}$
\end{tabular}

Parameters of the model used in all the simulations.

The variation of the input, expected with the addition of stimuli signal, has a high effect in the relay nuclei activity $\phi_{s}^{(0)}$, and a less considerable effect in the reticular nucleus activity $\phi_{r}^{(0)}$, and the cortical activity $\phi_{e}^{(0)}$. Fig $1 \mathrm{D}$ displays the relative changes

121 122 123 
of the loop-gains and the steady-state of the neural populations for the changes of the steady-state input activity.

Fig 1. Selection of baseline simulation parameters. (A) Trajectories of parameters searching in the XYZ-space. Selected parameters point indicated with a black diamond. The change of the steady-state input $\phi_{n}^{(0)}$ does not affect the X-axis value. (B) Dynamics of the model for parameters in the linear interpolation between N2 and N3 sleep stages with $\phi_{n}^{(0)}=0$. The selected point of parameters is closer to the N3 stage, keeping some additional energy in the SP band compared to the N3 published parameters 25. (C) Dynamics of the model while changing $\phi_{n}^{(0)}$ in the selected point of simulation at the interpolation trajectory. (D) Relative changes with respect to $\phi_{n}^{(0)}=1$ of the loop gains and the neural population's steady-state response values for the same cases of panel C. The loop-gain with more change is $G_{e s} G_{r e} G_{s r}$. The change of $\phi_{e}^{(0)}$, $G_{\mathrm{ee}}$, and $\mathrm{G}_{\mathrm{ei}}$ are similar and they are the less sensitive to the variation of $\phi_{n}^{(0)}$

\section{Enhancement of SWS events on the eirs-NFT model}

We searched for changes in the occurrence of the slow-wave sleep events (SO and SP) when we added stimulation $u(t)$ to the noisy input propagation field $\phi_{n}(\mathbf{r}, t)$. Fig $2 \mathrm{~A}$ shows the simulated cortical activity for three different stimulation types: random (STIM-R), periodic (STIM-P), and closed-loop with a target-phase (STIM-CL). The top panel shows the simulated cortical activity for the SHAM condition, $b(t)$. STIM-R stimulation delivers stimuli pulses at random times from a Poisson stochastic process with a mean inter-stimulus time. With STIM-P, the pulses occur at a fixed inter-stimulus period. STIM-CL follows the ongoing activity at a narrow band delivering a stimulus every time the signal arrives at a target-phase (see below, section SWS events activity variation with closed-loop stimulation). Fig $2 \mathrm{~B}$ shows the correspondent scalograms for each time series in Fig 2 A, where $\delta$-band activity $(0.5-4$ $\mathrm{Hz}$ ) - that includes SO-band $(0.5-1.25 \mathrm{~Hz})$ - is present through all the time. On the other hand, the SP-band activity $(9-16 \mathrm{~Hz})$ occurs at discrete periods, some of them with enough duration and amplitude to be classified as spindles.

Fig $2 \mathrm{C}$ focuses on a smaller time window when two SPs and one SO occur. The vertical marks in Figs $2 \mathrm{~A}$ and $2 \mathrm{C}$ show the start time point of each stimulus pulse, that in this case consist of decreasing ramps of $0.1 \mathrm{~s}$ duration and 40 a.u. of energy. The horizontal marks in Figs 2/A and 2 $\mathrm{C}$ represent detected SWS events.

Using the time-averaged scalograms, we calculated the spectrums shown in Fig $2 \mathrm{D}$. We define the power difference index, $I^{(S O, S P)}$, as the area between the corresponding STIM condition and SHAM spectrums inside the frequency band of interest, normalized by the sum of the power of both cases. Then, $I^{(S O, S P)}$ quantifies the differences with respect to the SHAM case for the frequency bands of interest (see Methods).

\section{Selection of the stimulus pulse characteristics with open-loop stimulation}

We characterized the efficiency of different stimulation signals to enhance the occurrence of slow-wave events. In a first step, we tested stimulus pulse features with the open-loop stimulation (STIM-R and STIM-P). We looked at power differences at the frequency bands of interest and the number of occurrences of SWS events while changing the shape, the energy, and the duration of the stimulus pulse. In the following, we will evaluate each of these parameters' impact on the co-occurrence of SP and SOs. 
Fig 2. Excerpt of Simulations (A) Excerpts from the time series of the stimulation. From top to bottom, SHAM, STIM-R, STIM-P, and STIM-CL simulations. Vertical bars show stimuli delivery. The detected events above each time series correspond to SOs, and the detected SPs are below. The dots in the marks of detected events represent intervals of 0.5 seconds. The stimulation parameters are: pulse shape: decreasing ramp, pulse energy $=40$ (a. u.), duration $=0.1 \mathrm{~s}$, target-phase of closed-loop $=45$ degrees, closed-loop (STIM-CL) and periodic (STIM-P) stimulation frequency $=0.85 \mathrm{~Hz}$, aleatory stimulation (STIM-R) times come from a Poisson distribution with $\lambda=1 / 0.85 \mathrm{~s}$. (B) Wavelet scalograms of SHAM, STIM-R, STIM-P, and STIM-CL activity shown in (A). The increase of the SO-power is visible in the scalograms, and the increase of both SWS events occurrence is notable with their detection in the time domain. (C) Zoom of the box of STIM-CL time series. Top, z-score of $x(t)$, and the detected SWS events. Middle, signal filtered at the event-bands and at $0.85 \mathrm{~Hz}$ for phase detection in STIM-CL. Bottom, comparison of the online detected phase and the offline phase got by Hilbert transform. (D) Wavelet spectrums calculated by the time average of the scalograms in (B). Note that the shadowed area between the curves STIM-CL and SHAM is the numerator in the calculation of $I^{(S O)}$ for STIM-CL (Eq (8)).

\section{Changes on SWS events by pulse shape}

With STIM-P and STIM-R, the shape, duration, and pulse energy change the temporal and frequency domain characteristics of $u(t)$. The stimulation frequency also modifies them in the case of STIM-P. We tested for the six shapes in Table 2 , and shown in Figs $3 \mathrm{~A}-\mathrm{B}$, with the same duration (0.1 seconds), keeping constant the stimulus pulse energy ( $E_{p}=40$ a. u.) and the stimulation frequency ( 0.2 pulses per second). Fig $3 \mathrm{C}$ shows changes in the SO, and Fig $3 \mathrm{D}$ shows changes about the SPs. The power difference index $\left(x\right.$-axis: $\left.I^{(S O)}, I^{(S P)}\right)$ and the number of occurrences $(y$-axis: $N_{S O} /$ min., $N_{S P} /$ min.) are plotted simultaneously for each type of SWS event. The decreasing ramp overpasses the results of all other shapes in both events and both measurements with significant differences (Welch's t-test, $p<0.01$ ).

Table 2. Stimulus pulse shapes

\begin{tabular}{l|l|l} 
Shape & Energy $E_{\text {pulse }}$ & Stim. Amplitude \\
Rectangular & $A^{2} D$ & $\mathrm{~A}=20.00$ \\
Gaussian & $0.3957 A^{2} D$ & $\mathrm{~A}=31.79$ \\
Half trapece & $2 A^{2} D / 3$ & $\mathrm{~A}=24.56$ \\
Triangular & $A^{2} D / 3$ & $\mathrm{~A}=34.64$ \\
Rising ramp & $A^{2} D / 3$ & $\mathrm{~A}=34.64$ \\
Decreasing ramp & $A^{2} D / 3$ & $\mathrm{~A}=34.64$
\end{tabular}

The co-occurrence of $S P$ and $S O$ is evaluated using: (i) the conditional probability of counted co-occurrences given the number of detected spindles, $P(C \mid S P)$; and (ii) the probability of slow oscillations, $P(S O)$. In Fig $3 \mathrm{E}$, a positive result means that $P(C \mid S P)$ is higher than $P(S O)$; in other words, that the probability of spindles co-occurring with SOs is higher than chance. Given this, both ramp shapes (decreasing and increasing) have positive results for the co-occurrence of events.

\section{Changes on SWS events by duration and energy of stimulus pulse}

Other stimulus pulse characteristics could modify the SWS events measurements. The pulse duration modifies the influence of stimulation on the frequency domain (narrower stimulus, wider spectral response), and changes in $I^{(S O)}$ and $I^{(S P)}$ are expected from rising the pulse energy. We tested for different pulse duration keeping constant the 
Fig 3. Stimulation results changing the shape of the stimulus pulse with rhythmic stimulation. (A) The tested pulse shapes are the decreasing ramp (1), trapeze (2), Gaussian (3); (B) rectangular (4), triangular (5), and rising ramp (6). These panels show the pulse shapes with the same $E_{p}$. (C) Changes in SOs occurrence by stimulus shape. (D) Changes in SPs occurrence by stimulus shape. (E) Changes in the co-occurrence of both SWS events by stimulus shape. The identity-diagonal in (E) represents the chance to explain the temporal coincidences by the time percentage of SO occurrence

pulse energy (40 a. u., rectangular pulses) Figs 4 A-C, and for various pulse energies keeping constant the duration (0.1 s, decreasing ramp pulses) Figs $4 \mathrm{D}-\mathrm{F}$, both by changing the pulse amplitude.

Among the tested duration values, the $0.05 \mathrm{~s}$ pulse displays better results for all measurements with statistical significance $(p<0.01)$, except in $N_{S P} /$ min. vs. $0.075 \mathrm{~s}$ pulses $(p>0.03)$.

The minimum pulse energy was selected to overpass the input-noise power of $9.67 \mathrm{~s}^{-2}$ per second in each node. $I^{(S O)}$ and $I^{(S P)}$ increase as the pulse energy rises with statistical differences at each increase step $(p<0.01)$, but the energy 100 (a. u.) is not different from 80 (a. u.) for $N_{S O} / \mathrm{min}$. and $N_{S P} / \min$. $(p>0.08)$. The minimum energy 10 (a. u.) is not different from SHAM in $N_{S 0} /$ min. and $N_{S P} /$ min. $(p>0.1)$. Interestingly, Fig $4 \mathrm{D}$ shows a peak of $N_{S O} / \mathrm{min}$. at 60 (a. u.), but without statistical difference from their neighboring pulse energies. Regarding the co-occurrence of events, the rectangular pulse shape of $0.05 \mathrm{~s}$, and the decreasing ramp pulses of $0.1 \mathrm{~s}$ with energies above 40 (a. u.) show positive results in Figs $4 \mathrm{C}$ and $4 \mathrm{~F}$, respectively.

The overall results of Fig 4 suggest that the best choice is the $0.05 \mathrm{~s}$ duration pulse, always considering that lower duration translates to higher amplitude in order to keep the same pulse energy, which in turn must be enough to overpass the intrinsic activity. Nevertheless, optimal results are found without need to rise over a six-fold value of the intrinsic power per second.

When exploring the stimulation frequency using a decreasing ramp of 0.1 seconds, we found no statistical differences between frequencies in the 0.5-1.25 Hz range (see S1 Fig). Stimulation with pulses at random intervals (STIM-R) has very similar effects of periodic pulses (STIM-P) S2 Fig, except for a higher $N_{S O} / \mathrm{min}$. with STIM-P than STIM-R (see the first row of Fig 4 and the second row of S2 Fig). Nevertheless, the results keep the same order between the characteristics variation. Thus, the difference of effects in Fig 4 comes from the pulse characteristics and not from the stimulation periodicity.

Fig 4. Stimulation results changing one parameter of the stimulus pulse with rhythmic stimulation. Top:(A) Changes in SOs by pulse duration. (B) Changes in SPs by pulse duration. (C) Changes in the co-occurrence of both SWS events by pulse duration. Bottom: (D) Changes in SOs by pulse energy. (E) Changes in SPs by pulse energy. (F) Changes in the co-occurrence of both SWS events by pulse energy. Solid black lines are the average values of SHAM condition, and dashed lines indicate one standard deviation. The identity-diagonal in $(\mathrm{C})$, and $(\mathrm{F})$ represents the chance to explain the temporal coincidences just by the time percentage of SO occurrence. 


\section{SWS events activity variation with closed-loop stimulation}

Next, we asked whether the phase of the ongoing activity could influence the power and the number of slow-wave sleep events at the moment of the stimulus onset. To answer this, we implemented a closed-loop stimulation protocol. Here, the stimulus pulse is applied when the inverse-notch filtered signal arrives at a particular target phase (see Fig 5A and Methods). Our phase detector used a fixed frequency of the ongoing activity, and $0.85 \mathrm{~Hz}$ has been used in other closed-loop implementation as the central frequency of a phase-locked loop (PLL) stage [28,29].

We applied the decreasing ramp stimulation pulses of $0.1 \mathrm{~s}$ duration and $E_{p}=40$ (a. u.) in the three stimulation cases: STIM-P, STIM-R, and STIM-CL. STIM-CL was further divided into STIM-CL 0 (stimulus onset when the phase of slow oscillations arrives at 0 degrees; the upside cross-zero point), STIM-CL 45 (45 degrees) and STIM-CL 90 (90 degrees; up-peak of the slow oscillation).

The STIM-CL 0 case overpasses the other two closed-loop stimulations in all the measurements as shown in Fig $5 \mathrm{~B}-\mathrm{D}$, with statistical significance in $I^{(S P)}$ and $P(S O)$ $(p<0.01$ Welch's independent t-test if both cases overpass Shapiro, otherwise Wilcoxon test, see S3 Table and S4 Table for all t-values and p-values). The STIM-CL 0 case is also statistically different from STIM-CL 90 for $I^{(S O)}$.

In relation to the open-loop stimulation cases, STIM-CL 0 overpasses STIM-P and STIM-R in $I^{(S O)}\left(p<0.01\right.$ vs STIM-P), $I^{(S P)}\left(p<0.01\right.$ vs STIM-R) and $N_{S P} / \mathrm{min} .$. Interestingly, STIM-P overpasses all other stimulations in $N_{S O} / \mathrm{min}$. but is only statistically different with STIM-R. These results probably come from the zero chance in STIM-P of two pulses occurring with an inter-pulse interval less than the stimulation frequency period.

The co-occurrence of events shows positive results in Fig 5D for all stimulation cases. STIM-P and STIM-CL 0 are very similar in the $P(S O)$ versus $P(C \mid S P)$ plane, but this plot does not say anything about the timing of occurrence of these temporal coincidences.

Fig 5. Closed-loop stimulation driver and results with different stimulation type. (A) Diagram of the closed-loop driver. The phase of a specific frequency from output signal $x(t)$ triggers the pulse generator when it arrives at the target-phase. At the bottom, changes in SWS events at a desired phase closed-loop stimulation, STIM-P, and STIM-R. (B) Changes in the SOs number of events and power index $I^{(S O)}$. (C) Changes in the SPs number of events and power index $I^{(S P)}$. (D) Changes in the co-occurrence of both events. The identity-diagonal represents the chance to explain the temporal coincidences just by the time percentage of $\mathrm{SO}$ occurrence.

\section{Timing of SWS events occurrence}

A higher occurrence, or higher power of slow-wave events, may not be enough to enhance memory consolidation 30. The relative timing of occurrence between SPs and SOs also play a role, with SPs being expected to be nested in the up-phase of a slow oscillation. Fig 6 shows histograms of the time delays between the SO's down-peak and the center of the SP from coincident events. Fig 6 also displays the average amplitude of the SOs, taken from $x(t)$, showing how the timing of the centers of the SPs are related to the down- and up-phase of SOs.

The histograms in Fig 6 include all coincident events from the total simulation time per stimulation condition. All stimulation conditions produce more SPs at the up-peak of SOs. Moreover, STIM-CL 90 condition is statistically different to the STIM-CL 0 and SHAM cases (Kolmogorv-Smirnov test, $p<0.05$, see Tab 3). SPs' timing 


\begin{tabular}{l|rrrrrr} 
& SHAM & STIM-R & STIM-P & STIM-CL 0 & STIM-CL 45 & STIM-CL 90 \\
\hline Co-occurrences & 77 & 267 & 304 & 317 & 267 & 263 \\
\hline SHAM & - & $* 0.011$ & 0.116 & 0.173 & $* * 0.008$ & $* 0.015$ \\
STIM-R & $* 0.011$ & - & 0.115 & $* 0.032$ & 0.509 & 0.868 \\
STIM-P & 0.116 & 0.115 & - & 0.840 & 0.367 & 0.116 \\
STIM-CL 0 & 0.173 & $* 0.032$ & 0.840 & - & 0.137 & $* 0.026$ \\
STIM-CL 45 & $* * 0.008$ & 0.509 & 0.367 & 0.137 & - & 0.638 \\
STIM-CL 90 & $* 0.015$ & 0.868 & 0.116 & $* 0.026$ & 0.638 & -
\end{tabular}

Table 3. Kolmogorov-Smirnov test p-values from searching for dissimilarity on the distribution of time-delay from the down-peak of the coincident slow oscillations and spindles. The mark ${ }^{\prime}{ }^{\prime}$ ' indicates $p<0.05$, and ${ }^{* *}$ ' indicates $p<0.01$.

distribution from STIM-CL 45 is also significantly different from the SHAM distribution $(p<0.01)$. Other significant differences $(p<0.05)$ are STIM-R vs. SHAM, and STIM-R vs. STIM-CL 0. The STIM-CL 45 and STIM-CL 90 show better SPs' timing, but the co-occurred events and the other measurements are higher with STIM-CL 0. Then, STIM-CL 0 is the best among the tested cases of stimulation.

Fig 6. SO down-peak locked results with different stimulation type.

Changes in the temporal occurrence of SWS events at the three closed-loop stimulation conditions. The histograms of spindles occurrences consider the entire simulation time per each stimulation type. The left vertical scale indicates the number of spindles with the center at the time delay from the SO down-peak indicated in the horizontal axis. The displayed average come from all detected SOs and all simulations using a decreasing ramp pulse, with pulse energy $=40$ (a. u.), duration $=0.1 \mathrm{~s}$, stimulation frequency and central frequency for phase extraction $=0.85 \mathrm{~Hz}$. In each stimulation condition, 900 pulses were delivered.

\section{Discussion}

\section{Selection of stimulation parameters}

This research asks which stimulation pattern is effective in raising the power of slow-wave sleep events, consequently increasing the SP and SO occurrences. Moreover, we were also interested in maximizing the co-occurrence of SP with the up-peak of SO.

Our most significant result is that a pulse of 0.05 seconds with decreasing ramp shape maximizes the power and number of both SWS events. The features of the stimulation pulses delivered with STIM-P that result in higher $I^{(S O)}, I^{(S P)}, N_{S O} / \mathrm{min}$., and $N_{S P} /$ min. are also above the diagonal of chance in the plane $P(C \mid S P)$ vs $P(S O)$. It is interesting to note that in experimental acoustic stimulation studies, the pulse duration was $50 \mathrm{~ms}$ [3, 28, 31, a right choice following our results for pulse duration.

The pulse energy must be higher than the intrinsic activity. Following our results, the preferred energy value is 60 (a. u.). However, the translation of this energy value to a physical measurement of sensory stimulation is difficult. Thus, the useful recommendation is to use an energy value around six times the intrinsic EEG activity power per second.

To achieve SPs' precise timing at the up-peak of SOs, STIM-CL 45 and STIM-CL 90 conditions significantly differ in the timing distribution compared with the SHAM condition. However, the STIM-CL 0 condition provides a better result in power and number of events than STIM-P except in $N_{S O} /$ min. as shown in Fig $5 \mathrm{~B}$, and with the higher number of co-occurrences indicated at Tab 3 
The shape of the stimulation pulses has small relevance in the previous neuro-stimulation works 32]. One probable cause is the technical difficulty of performing the stimulation following a particular shape-wave with precision in short duration times. For example, the triangular shape in Fig $3 \mathrm{~A}$ and the Gaussian shape in Fig $3 \mathrm{~B}$ may be indistinguishable from each other if the stimulation device has a small sampling rate. At the cellular level, the work of Pyragas et al. 33. demonstrates that using Pontryagin's maximum principle (a control theory formulation), a bipolar bang-off-bang waveform is optimal for the entrainment of a neuron to a specific frequency. This waveform type is not suitable for sensory stimulation, because a sensory stimulus affects excitatory and inhibitory neuronal populations at the same time [34. With basis on the receptive fields of the visual perception system and with the assumption of the stimulus independence from the constant variance Gaussian noise input [35], we used a difference of Gaussians as a spatial filter of the simulated sensory input.

Different pulse shapes could generate benefits for stimulating during sleep, like soft slopes for avoiding abrupt sensory inputs of high prediction error and contrast-dependent temporal dynamics. A sudden stimulus is detrimental in sleep by evoking non-desired arousal. However, the results for inducing SPs and SOs were better when each pulse began with a high amplitude (see also S6 Fig). The decreasing ramp shapes produce more beneficial and notorious changes for both SWS events. In the same direction, small duration pulses with higher initial amplitude for keeping the same pulse energy showed a higher increase in the power of both SWS events bands and a higher $N_{S P} /$ min. in Fig $4 \mathrm{~B}$. Furthermore, the rising ramp (time-mirrored version of the decreasing ramp) results in Fig $3 \mathrm{D}$ have the lowest performance of the tested shapes. These results suggest high relevance of the onset amplitude and the intrinsic phase of the stimulus pulse. Secondary effects of these stimuli, such as non-desired arousal, will have to be experimentally determined.

\section{Results-driven by the model dynamic linear and non-linear effects}

Mean-field models of the brain activity relay in two assumptions: The average population dynamics are similar to one neuron dynamics, and the average is enough statistic to characterize the population activity 22,36. Further, the output activity of a mean-field neural population depends on the dynamics (see Methods Eq (1)) and statistics of the incoming signals (Eq 4). In addition to the $u(t)$ dynamics, the input activity's statistics could play a role on the output dynamics of the model.

As in previous works of the Neural Field Theory [23 25, 27], we used Gaussian white noise as input, which provides the linear impulse response of the model. The noisy input activity represents the net contribution from other sources as the basal ganglia, brainstem, and peripheral nervous system [25]. At the minute scale, variations from the average input value come from these region's activity rather than modifications to the connection's strengths.In our simulations, we used a permanent average value for the noisy input.

We superimpose a pulse train in this approach, modifying the input mean value, and its variance. Still, the steady-state results in Fig $1 \mathrm{C}$ do not predict all the dynamic effects produced by adding a pulse train at the model's input.

The most notable difference from the expected results with the steady-state approximated response appears when the tested stimulation parameter is the pulse energy. Figs $4 \mathrm{D}$ and $4 \mathrm{E}$ show that with the rise of pulses energy, there is an increase in the power of both SWS events bands. The increase of $I^{(S O)}$ is opposite to the expected 
result from Fig $1 \mathrm{C}$ when the steady-state input rises. This increase in power does not come only from the periodicity of stimulation. The aleatory stimulation also increases the event's bands' power similarly (see S2 Fig). Moreover, in conjunction with results in S1 Fig, a specific stimulation frequency may not be too relevant for enhancing the power of both SWS events. It is also important to note that there are no significant differences in the results between STIM-R simulations.

Another possible way to change the input signal's statistics, and consequently its steady-state value, is by modifying their spatial characteristics. The coupling with sensory regions could matter to get results and predictions with sensory stimulation. However, the nodes' position in our model does not have a particular relation with a cortical region. Results from harmonics studies [37, 38 make it possible to assign a physiologically-related topography in future works. Consequently, the results reported here are not related to a particular sensory type; they are general in how the increase of activity in a delimited region could modify the whole system's output.

More work is needed to consider non-linear effects that surpass the expected response using the linear approximation to explain the obtained results with stimulation. The use of modern control techniques [39], and the integration of information theory measurements [40] could provide a better analytical approximation of the results than traditional linear systems analysis.

\section{Relation to similar computational works}

The work of Schellenberger et al. 41] tries to reproduce in a neural-mass model the experimental results from Ngo et al. 3. with closed-loop stimulation. They recover the experimental results using two external calcium-dependent currents and an anomalous rectifier current responsible for the waxing and waning structure of the spindle oscillations. The authors employed the same neural populations of eirs-NFT with one additional connection, the auto-connection of the thalamic reticular nucleus, and two additional noise inputs to the cortical populations. Similar to our approach, the sensory stimulation ( $80 \mathrm{~ms}$ square pulses with amplitude of $70 \mathrm{~s}^{-1}$ ) is added to the noise input of the relay nuclei. The simulation results show high similarity with the SO band's experimental ones on amplitude and timing, but differences in the SP band activity's timing.

Wei et al. 21, 42, 43 tested several ways of closed-loop stimulation, including the protocol of Ngo et al. 3], in a cellular level model. Their results showed that the correct spatio-temporal localization of stimuli could facilitate the replay of a cortical sequence associated with the stimulation site, pointing to the need for peaks nesting for strengthening the neural connections. For this purpose, they propose stimuli application at the end of the SO's down-state, near the start of the SO up-state $\left(0^{\mathrm{O}}\right.$ by sine reference), almost matching with our stimulation target-phase proposal.

\section{Relation with experimental works and further work}

Non-invasive methods of neural system stimulation operate at large spatial scales, and they can be by direct application of electrical or magnetic fields or by the use of sensory stimuli to modify neural activity 32,44 . The non-invasive techniques are under active-research because of the potential clinical applications in humans. Several works 12,13 tried to enhance the SWS activity to achieve memory improvements. There were applications of olfactory [45, 46], auditory [3, 28], and tactile stimuli [47] with similar or even better results than direct stimulation $48[54]$.

Following the discoveries from previous works of Ngo et al., the application of stimulus at a precise phase of the ongoing cortical activity 3 obtains better behavioral results than rhythmic stimulation 31,55. Another result of the same authors show that 
the application of more than two consecutive pulses does not improve physiological outcomes 56. These results suggest the presence of mechanisms that prevent an over-driving of SO activity, which could be present in our case given a similar increase of $I^{(S O)}$ but lesser $N_{S O} / \mathrm{min}$. with STIM-R (see S1 Fig). A recent work 30 shows that this closed-loop stimulation pattern does not always enhance memory consolidation even with the increase of the power of the SWS events.

Our results suggest that the shape, energy, and application phase of the stimulation pulses modifies the power and occurrence of SWS events. On the framework of EEG, these stimuli parameters are often neglected in sensory event-related potential (ERP) analysis, given the confounds with attention in awake experimental studies [57] and the incomplete knowledge about the codification pathways of sensory information. However, these stimulation characteristics are under research for neural implants, and direct stimulation [58. Finally, the selected stimulation parameters need experimental verification to correlate with memory task performance.

\section{Conclusion}

The neural field model can reproduce SWS activity with the occurrence of slow oscillations and sleep spindles. Rhythmic, aleatory, and closed-loop stimulation was applied using this model to enhance the SWS events. Closed-loop stimulation, with stimulus applied at zero degrees as target phase of the ongoing activity filtered at a specific frequency, increases the power and the number of both SWS events, together with their co-occurrences. Also, closed-loop stimulation at other target-phases changes spindles' timing in respect to the down-peak of slow oscillations. Our results indicate that the number of slow oscillations decreases when the stimulation pulse energy overpass more than six times the background noise power per second. On the other hand, increasing the stimulus energy increases the SPs' power and their number of occurrences. The stimulus pulse's promoted parameters to get a higher occurrence of both events is the decreasing ramp shape with a duration of $50 \mathrm{~ms}$ applied at target-phase zero of closed-loop stimulation.

\section{Materials and methods}

\section{Large-scale brain model: Neural Field Theory}

Neural field theory represents the the activity of a whole population of neurons as its mean firing rate. Spatial propagation can then be calculated to obtain the spatio-temporal evolution of neuronal activity 22 .

Thalamo-cortical interactions can be modeled using Neural Field Theory, where both cortical and thalamic regions are each represented by a pair of excitatory and inhibitory neural populations. The populations relate to known structures. In the cortex, the excitatory population $e$ corresponds to the pyramidal neurons, while inhibitory population $i$ represents the inter-neurons. In the thalamus, the excitatory population represents the relay nucleus $s$ of incoming sensory information. Similarly, the thalamic inhibitory population represents the reticular nucleus $r$. Fig $7 \mathrm{~A}$ schematizes the connections between the four populations in the eirs-NFT model.

The dynamics of the mean soma voltage, $V_{a}$, of the neural population $a$, with incoming activity from another $b \in \mathbf{b}$ population, is represented as (see also Fig7 $\mathrm{B}$ )

$$
\frac{1}{\alpha \beta} \frac{d^{2} V_{a}(\mathbf{r}, t)}{d t^{2}}(\mathbf{r}, t)+\left(\frac{1}{\alpha}+\frac{1}{\beta}\right) \frac{d V_{a}(\mathbf{r}, t)}{d t}+V_{a}(\mathbf{r}, t)=\sum_{\mathbf{b}} \nu_{a b} \phi_{b}\left(\mathbf{r}, t-\tau_{a b}\right),
$$


where $\alpha$ is the inverse of the decaying time of the impulse response, and $\beta$ is the inverse of the rising time of the impulse response. Both parameters shape the

post-synapto-dendritic response. $\nu_{a b}$ is the strength of the connection from population $b$ to $a$, with $a, b \in \mathbf{b}=\{e, i, r, s\}$, and its sign determines whether the connection is excitatory (positive connection strength) or inhibitory (negative connection strength). $\tau_{a b}$ is the propagation delay between the populations $a$ and $b$. Then, the model uses a sigmoid function to convert the soma voltage into the firing rate population response, $Q_{a}$, as follows

$$
Q_{a}(\mathbf{r}, t)=S\left[V_{a}(\mathbf{r}, t)\right]=\frac{Q_{\max }}{1+\exp \left(-\left(V_{a}(\mathbf{r}, t)-\theta\right) / \sigma_{\rho}\right)},
$$

where $Q_{\max }$ is the maximum firing response, and the sigmoid function approximates the normal cumulative probability distribution of the firing threshold voltage with mean $\theta$ and standard deviation $\sigma$ where $\sigma_{\rho}=\sigma \sqrt{3} / \pi$.

The spatial propagation of the firing response only applies to the excitatory population $e$, the one with axons long enough for making it relevant 23. It is expressed as

$$
\frac{1}{\gamma_{e}^{2}} \frac{\partial^{2} \phi_{e}(\mathbf{r}, t)}{\partial t^{2}}+\frac{2}{\gamma_{e}} \frac{\partial \phi_{e}(\mathbf{r}, t)}{\partial t}+\phi_{e}(\mathbf{r}, t)-r_{e}^{2} \nabla^{2} \phi_{e}(\mathbf{r}, t)=Q_{e}(\mathbf{r}, t)
$$

where $\gamma_{e}=v_{e} / r_{e}$ is the temporal damping coefficient for pulses propagating at a velocity $v_{e}$ through axons with a characteristic range $r_{e}$. For the other populations, $\phi_{a}(\mathbf{r}, t)=Q_{a}(\mathbf{r}, t)$.

The expressions Eq (1)-Eq (3) govern the dynamics of each neural population. Their parameters are physiologically related values 23 and are listed in Table 1 . Note that the sub-indexes of the parameters follow the order: 'input to'- 'output from'. Fig7 8 represents the connections inside a population and relationship between the variables.

Fig 7. Neural Field Theory model and additional modules. (A) eirs-NFT model connections and loops. Arrow ended connections are excitatory, and dot ended connections are inhibitory. There are two intra-cortical loops with gains $G_{e e}$ and $G_{e i}$ related to the X-dimension, one intra-thalamic loop with gain $G_{s r} G_{r s}$ related to the Y-dimension, and the gains $G_{e s} G_{s e}$ and $G_{e s} G_{r e} G_{s r}$ are cortico-thalamic gain-loops related to the Z-dimension. (B) Diagram of the dynamics of a population of the Neural Field Theory. Particularly, the diagram shows as example the excitatory population $e$ of the eirs-NFT model. (C) Complete simulation diagram including the modules of spatial coupling of the sensory stimulation input and the spatial averaging at the output. The grid in the cortico-thalamic activity block shows the nodes distribution, and the application location of stimuli.

\section{Linear transfer function and loop-gains}

To calculate the spectrum of the system's linear approximation with diverse sets of connections strengths, we used the steady-state solutions of the Eq (1) and Eq (3). The steady-state solution of the corticothalamic model comes from setting all temporal and spatial derivatives to zero. Defining $\phi_{e}^{(0)}$ and $\phi_{n}^{(0)}$ as the steady-state value of the cortical neural population and the input, respectively, the steady-state solution 24 is

$$
\begin{aligned}
& S^{-1}\left[\phi_{e}^{(0)}\right]-\left(\nu_{e e}+\nu_{e i}\right) \phi_{e}^{(0)}= \\
& \nu_{e s} S\left[\nu_{s e} \phi_{e}^{(0)}+\nu_{s r} S\left[\nu_{r e} \phi_{e}^{(0)}+\left(\nu_{r s} / \nu_{e s}\right)\left(S^{-1}\left[\phi_{e}^{(0)}\right]-\left(\nu_{e e}+\nu_{e i}\right) \phi_{e}^{(0)}\right)\right]+\nu_{s n} \phi_{n}^{(0)}\right] .
\end{aligned}
$$


We searched the steady-state solutions that accomplish Eq (4) using the function fsolve of Matlab with an initial value for $\phi_{e}^{(0)}=10 \mathrm{~s}^{-1}$.

The linear approximation of the sigmoid function in Eq (2) uses its Taylor's serie expansion

$$
Q_{a}(\mathbf{r}, t)=Q_{a}^{(0)}+\rho_{a}\left(V_{a}(\mathbf{r}, t)-V_{a}^{(0)}\right)+O^{2}
$$

where $\rho_{a}$ is the slope of the sigmoid function evaluated at $V_{a}^{(0)}$, the steady-state soma voltage of the population $a$. Note from Eq (3) that, in the steady-state, $\phi_{a}^{(0)}=Q_{a}^{(0)}$ and we can treat each variable of the model as the perturbation from their steady-state value, reducing the above expression to

$$
\phi_{a}(\mathbf{r}, t)=Q_{a}(\mathbf{r}, t)=\rho_{a} V_{a}(\mathbf{r}, t) .
$$

Taking the Fourier transform of Eq (1), Eq (3), and Eq (6), and rearranging and eliminating $\phi_{r}$ and $\phi_{s}$, leads to the system's spectrum with output $\phi_{e}$, and input $\phi_{n} 24,25$.

$$
\begin{gathered}
\frac{\phi_{e}(\mathbf{k}, \omega)}{\phi_{n}(\mathbf{k}, \omega)}=\frac{L^{2} G_{e s} G_{s n} \exp \left(i \omega t_{0} / 2\right)}{\left(1-L G_{e i}\right)\left(1-L^{2} G_{s r} G_{r s}\right)\left(\delta-\mathbf{k}^{2} r_{e}^{2}\right)}, \\
\delta=\left(1-\frac{i \omega}{\gamma_{e}}\right)^{2}-\frac{1}{1-L G_{e i}}\left[\frac{G_{e e} L+\left(G_{e s} G_{s e} L^{2}+G_{e s} G_{r e} G_{s r} L^{3}\right) \exp \left(i \omega t_{0}\right)}{1-L^{2} G_{s r} G_{r s}}\right],
\end{gathered}
$$

where $G_{a b}=\rho_{a} \nu_{a b}$ is the gain of the connection $a b$, and $L$ is an abbreviation for $L(\omega)$, the reciprocal of the Fourier transform of the operators applied to $V_{a}(\mathbf{r}, t)$ in Eq (1) and expressed as

$$
L(\omega)=\left(\left(1-\frac{i \omega}{\alpha}\right)\left(1-\frac{i \omega}{\beta}\right)\right)^{-1} .
$$

The linear approximation in Eq (7) uses a reduction of the model parameters with the same incoming connection strengths for the excitatory and inhibitory populations of the cortex, i. e. the connection strength $\nu_{e e}=\nu_{i e}, \nu_{e i}=\nu_{i i}$, and $\nu_{e s}=\nu_{i s}$. The gray arrows in Fig 7]A represents the gain loops in Eq (7); $G_{e i}$ becomes an additional loop gain with the reduction of parameters. Further, the non-zero propagation delays considered in Eq (7) only correspond to the ones between the corticothalamic and thalamo-cortical populations, $\tau_{e s}=\tau_{s e}=\tau_{r e}=t_{0} / 2$.

\section{Spatial coupling of stimuli input}

The eirs-NFT model uses spatio-temporal variables, but our stimuli input is a single-channel time series. To give the stimuli a spatial representation, the signal goes through the sensory input module at the left of Fig $7 \mathrm{C}$. We formed a receptive field using a Difference of Gaussians (DoG) spatial kernel:

$$
u^{\prime}(\mathbf{r}, t)=\frac{u(t)}{\sqrt{2 \pi} \sigma_{E}} \exp \left(\frac{\left(\mathbf{r}-\mu_{\mathbf{r}}\right)^{2}}{\sigma_{E}^{2}}\right)-\frac{u(t)}{\sqrt{2 \pi} \sigma_{I}} \exp \left(\frac{\left(\mathbf{r}-\mu_{\mathbf{r}}\right)^{2}}{\sigma_{I}^{2}}\right)
$$

The spatial filter is centered at an interior node $\mu_{\mathbf{r}}(x=7, y=7)$ of the square sheet of 256 nodes with positive standard deviation $\sigma_{E}=1$ and negative standard deviation $\sigma_{I}=2$. The chosen center and standard deviations values avoid the grid boundaries.

As shown in Fig $7 \mathrm{C}$, before entering the thalamo-cortical model, we added Gaussian white noise, $v(\mathbf{r}, t)$, forming the potential field of the $n$th population, $\phi_{n}(\mathbf{r}, t)$. 


\section{Simulation procedure}

We solved the model dynamics in Eq (1)-Eq (3) using the Euler's integration method with a time step $h=1 e-4$ seconds, and a uniform sheet of $N=256$ equidistant nodes in a square cortex of 0.5 meters per side.

Each simulation lasts 910 seconds. We performed five simulations for every tested stimulation pattern $u(t)$, each with a different random number generator seed, giving a total of 4550 seconds per stimulation case. The stimulation started after the first 5 seconds and stopped before the last 5 seconds. The initial conditions of neural populations variables come from a previous 6 -second simulation with initial conditions equal to zero, and without stimulation.

We stored the output cortical activity $\left.\phi_{e}(\mathbf{r}, t)\right)$, and the input signal $\phi_{n}(\mathbf{r}, t)$ with a sampling frequency of 100 samples per second.

We obtained an EEG-like signal, $x(t)$, spatially averaging the output activity for all time points, and then we subtracted its temporal mean value

$$
x(t)=\frac{1}{N} \sum_{\mathbf{r}} \phi_{e}(\mathbf{r}, t)-\bar{\phi}_{e}(\mathbf{r}, t),
$$

where $\mathbf{r}$ is the grid position, $N$ the number of nodes, and $\bar{\phi}_{e}(\mathbf{t}, t)$ the average of the cortical activity (see Fig $7 \mathrm{C}$, module at the right).

\section{Event detection in the time domain}

We detected the slow-wave sleep events (slow oscillations and sleep spindles) from the output signal of the complete model. In the following, we refer as $x(t)$ to the output signal of the stimulation cases (STIM), while $b(t)$ represents the output signal of the non-stimulation cases or SHAM condition.

\section{Detection of slow-oscillations events}

As our EEG-like signal, $x(t)$ is not in voltage units; then, we cannot use the classical method to detect SOs in EEG recordings. The classical method establishes SOs every time the signal has a negative peak below $-40 \mu \mathrm{V}$, and a peak-to-peak amplitude higher to $75 \mu \mathrm{V}$. As an alternative, we searched for events with time-lengths between 0.8 and 2 seconds (frequency: $0.5-1.25 \mathrm{~Hz}$ ) [3] , and we kept the higher amplitude oscillations .

The detection of SOs is based on the search for high amplitude oscillations in a delta-band sub-region. First, we filtered the signal in the SO frequency band (0.5-1.25 $\mathrm{Hz}$, Chebyshev type I filter, fourth-order, 1e-6dB allowed ripple, zero-phase lag). Second, we used the z-score of the filtered signal for searching the zero-crossing points. Later, we applied a threshold to the filtered signal, and we searched for negative peaks below the arbitrary low value of $-1 \mathrm{e}-06 \mathrm{~s}^{-1}$. Using the zero-crossing points, we calculated the peak-to-peak amplitude for the single oscillations that underpass the negative peak threshold. Finally, slow oscillations are confirmed if the peak-to-peak amplitude is higher than 1.25 times the baseline peak-to-peak amplitude average.

\section{Detection of sleep spindles}

Spindles are bursts of oscillations in the 9-16 Hz band. To detect them, we normalized each simulated output $x(t)$ by the mean and variance of their corresponding $b(t)$ signal. The results are single-channel time series expressed as z-scored EEG registers for each simulation. Then, we computed the root mean square (RMS) value of the z-score $x(t)$ filtered in the SPs frequency band (9-16 Hz, Chebyshev type I filter, fourth-order, 1e-6 dB allowed ripple, zero-phase lag) and applied an amplitude threshold for the 
automatic detection of SPs 18,59 . The RMS value calculated at each sample uses a 0.2 $\mathrm{s}$ window length, and we also smoothed it with a Hamming window of the same time length 60. SPs are then detected every time the signal overpasses a detection threshold of 1.25 standard deviations of the RMS value of the filtered baseline signal.

\section{Counting of co-occurrent events}

We defined co-occurrence of events every time SOs and SPs overlap at least 250ms. The counting process of co-occurrences uses tagged events to avoid duplication. We also extracted the time percentage of occurrence of SOs, SPs, and co-occurrences. From here, we use the identifier $N_{S O}$ for the number of slow oscillations, $N_{S P}$ for spindles, and $N_{C}$ for co-occurrence of events.

\section{Power increases in the event's frequency bands}

We define the scalogram power difference index $\left(I^{(j)}\right)$, which relates the power of the oscillations in the $j$ th frequency band as Eq (8)

$$
I^{(j)}=\frac{\sum_{s \in s_{j}} \sum_{t \in T}\left|S_{x}(s, t)\right|-\left|S_{b}(s, t)\right|}{\sum_{s \in s_{j}} \sum_{t \in T}\left|S_{x}(s, t)\right|+\left|S_{b}(s, t)\right|}, \quad j=S O, S P,
$$

where $S_{x}(s, t)$ is the STIM register scalogram, $S_{b}(s, t)$ is the baseline or SHAM register scalogram. $s_{S O}$ comprises the scales inside the $0.5-1.25 \mathrm{~Hz}$ spectrum related to slow oscillations and the $s_{S P}$ the range of scales correspondent to the frequency range of the sleep spindles of $9-16 \mathrm{~Hz} . T$ is the total simulation time.

We used the Morlet wavelet in the scalogram computation. The mother wavelet has central frequency $\omega_{0}=15$, using scaling factors, that result in a frequency resolution of 300 scales related to a starting frequency at 0.1 and ending at $30 \mathrm{~Hz}$. The scales are equally distanced in a logarithmic range 61.

\section{Closed-loop phase-lock driver}

The closed-loop stimulation requires the on-line detection of instantaneous phase of the ongoing activity, to apply stimuli at the target phase. For this, we filtered $x(t)$ with an IIR inverse-notch filter for $\omega_{0}=2 \pi f_{0} h$ with poles $z=\left\{0.9999 \exp \left( \pm j \omega_{0}\right), 0\right\}$, and zeros $z=\{-1,-1,1\}$, considering a scaling factor of $1 e-4$. In addition, we extracted the envelope of the filtered signal at each sample to normalize itself. The envelope detector (see Fig 5A in Results) has three stages: (i) We detected the peaks of the rectified signal using its absolute value. (ii) We exponentially decreased the peak value, with a decay rate of $12.5 \mathrm{~s}^{-1}$, until the detection of a new peak or the reaching of the limit of a sample counter. (iii) We normalized the filtered signal with the current envelope value at each sample.

On the normalized signal, $z(t)$, an additional IIR filter gets its $\pi / 2$ shifted-phase version $y(t)$. This shift-phase filter has the poles $z=\left\{0.9999 \exp \left( \pm j \omega_{0}\right), 0.999\right\}$, and zeros $z=\{-0.5 \exp ( \pm 0.495 \pi),-0.9\}$. The filter scaling factor is the half quotient of the sum of the denominator coefficients over the sum of the initial numerator coefficients.

The calculated atan $(y(t) / z(t))$ gets the phase in the range $(-\pi / 2, \pi / 2)$. We translated the phase to the range $(0,2 \pi)$ or $(0,360)$ using a cross-zero detector applied on the shift-phased signal.

The stimulus pulse generator is triggered when the phase arrives inside a hysteresis window of $\varepsilon= \pm 9$ degrees $( \pm 5 \%)$ around the desired target-phase. The trigger waits for the overpass of the phase value of 1.9 or 342 degrees before resetting for the next pulse applying. 
We applied the stimuli at a specific target-phase of a particular frequency in the closed-loop condition. The signal goes through a narrow band-pass filter and we detected the instantaneous phase with the arc-tangent with an online $\pi / 2$-shifted phase filtered version of the narrow band signal. We normalized the amplitude of the signal before the arc-tangent by the envelope amplitude. The envelope value came from a peak detector, where the peak value decreases exponentially with a decay rate of $12.5 \mathrm{~s}^{-1}$ until the detection of a new peak.

\section{Statistical tests}

We tested for normality of each set of results for each stimulation pattern with the Shapiro-Wilk test.

To compare between pairs of stimulation patterns following a normal distribution, we used the Welch's t-test to is the Welch's t-test. On the contrary, when at least one pattern does not follow a normal distribution, we used the Wilcoxon signed-rank test. We used the Kolmogorov-Smirnoff test for the similarity between the distributions of time delays between the down-peak of the slow oscillation and the center of the coincident spindle.

All the statistical analysis were implemented using the library scipy.stats 1.5 .2 in Python 3.7.

\section{Supporting information}

S1 Fig. Power and events changes by different stimulation frequency. (A) Changes by stimulation frequency on SO measurements. (B) Changes by stimulation frequency on spindles measurements. (C) Changes by stimulation frequency on probabilities of coincident events.(D) Changes by different mean $\lambda$ of aleatory stimulation on SO measurements. (E) Changes by different mean $\lambda$ of aleatory stimulation on spindles measurements. (F) Changes by different mean $\lambda$ of aleatory stimulation on probabilities of coincident events.

S2 Fig. Power and events changes by aleatory stimulation. Top:(A) Changes by shape in SO. (B) Changes by shape in spindles. (C) Changes in the probability of co-occurrence by shape. (D) Changes by pulse duration in SO. (E) Changes by pulse duration in spindles. (F) Changes by pulse duration in the probability of co-occurrence. (G) Changes by pulse energy in SO. (H) Changes by pulse energy in spindles. (I) Changes by pulse energy in the probability of co-occurrence.

S3 Table Welch's t-test of slow oscillations measurements. The t-values and p-values for changes in power and occurrence of slow oscillation events by different stimulation case. The symbol '*'indicates $p<0.01$, and 'w'indicates that one stimulation case doesn't accomplish the Shapiro test for normality.

S4 Table Welch's t-test of sleep spindles measurements. The t-values and $\mathrm{p}$-values for changes in power and occurrence of sleep spindles events by different stimulation case. The symbol '*'indicates $p<0.01$, and 'w'indicates that one stimulation case doesn't accomplish the Shapiro test for normality.

S5 Table Welch's t-test for $P(S O)$ and $P(C \mid S P)$. The t-values and p-values for changes in the probability of occurrence of slow oscillations and co-occurrences of events by different stimulation phase. Welch's t-test applied after the confirmation of 
normality by Shapiro test. The '*' indicates p-values $<0.01$. STIM-CL 0 number of spindles did not pass the normality test, and it neither did not pass the test with the SHAM condition, but is the highest value in the plot.

S6 Fig. Correlation of SWS events changes with the initial amplitude of the stimulation pulses. (A) Onset amplitude of stimulus vs $I^{(S O)}$. (B) Onset amplitude of stimulus vs slow oscillations. (C) Onset amplitude of stimulus vs probability of co-occurrence of events. (D) Onset amplitude of stimulus vs $I^{(S P)}$. (E) Onset amplitude of stimulus vs spindles. (F) Onset amplitude of stimulus vs conditional probability of co-occurrence respect to the occurrence of spindles.

\section{Acknowledgments}

This work was supported by CONICYT-Basal Project FB0008, CONICYT grant PhD scholarship No. 2118640, AFOSR Grant Nro. FA9550-19-1-0002, CNRS-PICS Nro. 07844 .

\section{References}

1. Dudai, Y., Karni, A., \& Born, J. The Consolidation and Transformation of Memory. Neuron. 2015; 88(1), 20-32.

https://doi.org/10.1016/j.neuron.2015.09.004

2. Sara, S. J. Sleep to Remember. The Journal of Neuroscience. 2017; 37(3), 457-463. https://doi.org/10.1523/JNEUROSCI.0297-16.2017

3. Ngo, H. V. V., Martinetz, T., Born, J., \& Mölle, M. Auditory closed-loop stimulation of the sleep slow oscillation enhances memory. Neuron. 2013; 78(3), 545-553. https://doi.org/10.1016/j.neuron.2013.03.006

4. Krishnan, G. P., Rosen, B. Q., Chen, J. Y., Muller, L., Sejnowski, T. J., Cash, S. S., Helgren. E., \& Bazhenov, M. Thalamocortical and intracortical laminar connectivity determines sleep spindle properties. PLoS Comp Bio. 2018; 14(6), 1-22. https://doi.org/10.1371/journal.pcbi.1006171

5. Jiang, X., Gonzalez-Martinez, J., \& Halgren, E. Coordination of Human Hippocampal Sharpwave Ripples during NREM Sleep with Cortical Theta Bursts, Spindles, Downstates, and Upstates. The Journal of Neuroscience : The Official Journal of the Society for Neuroscience. 2019; 39(44), 8744-8761. https://doi.org/10.1523/JNEUROSCI.2857-18.2019

6. Genzel, L., \& Wixted, J. T. Cellular and systems consolidation of declarative memory. In: Cognitive Neuroscience of Memory Consolidation; 2017. pp. 3-16.

7. Staresina, B. P., Bergmann, T. O., Bonnefond, M., Van Der Meij, R., Jensen, O., Deuker, L.,Elger, C. E., Axmacher, N., \& Fell, J. Hierarchical nesting of slow oscillations, spindles and ripples in the human hippocampus during sleep. Nature Neuroscience. 2015; 18(11), 1679-1686. https://doi.org/10.1038/nn.4119

8. Adamantidis, A. R., Gutierrez Herrera, C., \& Gent, T. C. Oscillating circuitries in the sleeping brain. Nature Reviews Neuroscience. 2019; Springer US. https://doi.org/10.1038/s41583-019-0223-4 
9. Antony, J. W., Schönauer, M., Staresina, B. P., \& Cairney, S. A. Sleep Spindles and Memory Reprocessing. Trends in Neurosciences. 2019; 42(1), 1-3. https://doi.org/10.1016/j.tins.2018.09.012

10. Geva-Sagiv, M., \& Nir, Y. Local sleep oscillations: Implications for memory consolidation. Frontiers in Neuroscience, 2019; 13(JUL), 1-7. https://doi.org/10.3389/fnins.2019.00813

11. Schönauer, M. Sleep Spindles: Timed for Memory Consolidation. Current Biology. 2018; 28(11), R656-R658. https://doi.org/10.1016/j.cub.2018.03.046

12. Cellini, N., \& Mednick, S. C. Stimulating the sleeping brain: Current approaches to modulating memory-related sleep physiology. Journal of Neuroscience Methods. 2018; 316, 125-136. https://doi.org/10.1016/j.jneumeth.2018.11.011

13. Hanslmayr, S., Axmacher, N., \& Inman, C. S. Modulating Human Memory via Entrainment of Brain Oscillations. Trends in Neurosciences. 2019; 1-15. https://doi.org/10.1016/j.tins.2019.04.004

14. Fernández-Ruiz, A., Oliva, A., de Oliveira, E. F., Rocha-Almeida, F., Tingley, D., \& Buzsáki, G. Long-duration hippocampal sharp wave ripples improve memory. Science. 2019; 364(6445), 1082-1086. https://doi.org/10.1126/science.aax0758

15. Fattinger, S., De Beukelaar, T. T., Ruddy, K. L., Volk, C., Heyse, N. C., Herbst, J. A., Hahnloser, R. H., Wenderoth, N. \& Huber, R. Deep sleep maintains learning efficiency of the human brain. Nature Communications. 2017; 8. https://doi.org/10.1038/ncomms15405

16. Leminen, M. M., Virkkala, J., Saure, E., Paajanen, T., Zee, P. C., Santostasi, t.G., Hublin, C., Müller, K., Porkka-Heiskanen, T., Huotilainen, M. \& Paunio, T. Enhanced memory consolidation via automatic sound stimulation during non-REM sleep. Sleep. 2017; 40(3). https://doi.org/10.1093/sleep/zsx003

17. Vosskuhl, J., Strüber, D., \& Herrmann, C. S. Non-invasive Brain Stimulation: A Paradigm Shift in Understanding Brain Oscillations. Frontiers in Human Neuroscience. 2018 12(May); 1-19. https://doi.org/10.3389/fnhum.2018.00211

18. Ngo, H. V. V., Seibold, M., Boche, D. C., Mölle, M., \& Born, J. Insights on auditory closed-loop stimulation targeting sleep spindles in slow oscillation up-states. Journal of Neuroscience Methods. 2018; 316, 117-124. https://doi.org/10.1016/j.jneumeth.2018.09.006

19. Batterink, L. J., Creery, J. D., \& Paller, K. A. Phase of Spontaneous Slow Oscillations during Sleep Influences Memory-Related Processing of Auditory Cues. Journal of Neuroscience. 2016; 36(4), 1401-1409. https://doi.org/10.1523/JNEUROSCI.3175-15.2016

20. Postnova, S. Sleep Modelling across Physiological Levels. Clocks \& Sleep. 2019; 1(1), 166-184. https://doi.org/10.3390/clockssleep1010015

21. Wei, Y., Krishnan, G. P., Marshall, L., Martinetz, T., \& Bazhenov, M. Stimulation Augments Spike Sequence Replay and Memory Consolidation during Slow-Wave Sleep. The Journal of Neuroscience. 2020; 40(4), 811-824. https://doi.org/10.1523/JNEUROSCI.1427-19.2019 
22. Sanz-Leon, P., Robinson, P. A., Knock, S. A., Drysdale, P. M., Abeysuriya, R. G., Fung, F. K., Rennie, C., \& Zhao, X. NFTsim: Theory and Simulation of Multiscale Neural Field Dynamics. PLOS Comp Bio. 2017; 14(8), 1-37. https://doi.org/10.1371/journal.pcbi.1006387

23. Robinson, P. A., Rennie, C. J., Rowe, D. L., \& O'Connor, C. Estimation of multiscale neurophysiologic parameters by electroencephalographic means. Human Brain Mapping. 2004; 23(1), 53-72. https://doi.org/10.1002/hbm.20032

24. Abeysuriya, R. G., Rennie, C. J., \& Robinson, P. A. Prediction and verification of nonlinear sleep spindle harmonic oscillations. Journal of Theoretical Biology. 2014; 344, 70-77. https://doi.org/10.1016/j.jtbi.2013.11.013

25. Abeysuriya, R. G., Rennie, C. J., \& Robinson, P. A. Physiologically based arousal state estimation and dynamics. Journal of Neuroscience Methods. 2015 Jun; 253, 55-69. https://doi.org/doi.org/10.1016/j.jneumeth.2015.06.002

26. Benbadis, S. R. Normal Sleep EEG. Medscape. 2016; 1-14. Retrieved from http://emedicine.medscape.com/article/1140322-overview\#showall

27. Roberts, J. A., \& Robinson, P. A. Corticothalamic dynamics: Structure of parameter space, spectra, instabilities, and reduced model. Physical Review E. 2012; 85(1), 1-20. https://doi.org/10.1103/PhysRevE.85.011910

28. Papalambros, N. A., Santostasi, G., Malkani, R. G. et al. Acoustic enhancement of sleep slow oscillations and concomitant memory improvement in older adults. Front. Hum .Neurosci., 2017, 1

29. Santostasi, G., Malkani, R., Riedner, B., Bellesi, M., Tononi, G., Paller, K. A., \& Zee, P. C. Phase-locked loop for precisely timed acoustic stimulation during sleep. J Neurosci Methods. 2016;259:101-114. doi:10.1016/j.jneumeth.2015.11.007

30. Henin, S., Borges, H., Shankar, A., Sarac, C., Melloni, L., Friedman, D., \& Liu, A. Closed-loop acoustic stimulation enhances sleep oscillations but not memory performance. ENeuro. 2019; 6(6). https://doi.org/10.1523/ENEURO.0306-19.2019

31. Ngo, H. V. V., Claussen, J. C., Born, J., \& Mölle, M. Induction of slow oscillations by rhythmic acoustic stimulation. Journal of Sleep Research. 2013; 22(1), 22-31. https://doi.org/10.1111/j.1365-2869.2012.01039.x

32. Kumar G., Ritt J. T. \& Ching S. Control theory for Closed Loop Neurophisiology Closed Loop Neuroscience. 2016; https://dx.doi.org/10.1016/B978-0-12*802452-2.00003-2

33. Pyragas, K., Fedaravičius, A. P., Pyragiene, T., \& Tass, P. A. Optimal waveform for entrainment of a spiking neuron with minimum stimulating charge. Physical Review E. 2018; 98(4), 1-14. https://doi.org/10.1103/PhysRevE.98.042216

34. Deco, G., Cruzat, J., Cabral, J., Tagliazucchi, E., Laufs, H., Logothetis, N. K., \& Kringelbach, M. L. Awakening: Predicting external stimulation to force transitions between different brain states. Proceedings of the National Academy of Sciences. 2019; 201905534. https://doi.org/10.1073/pnas.1905534116

35. Chalk, M., Masset, P., Gutkin, B., \& Deneve, S. Sensory noise predicts divisive reshaping of receptive fields. PLOS Comp Bio. 2017; 13(6), 1-26.

https://doi.org/10.1371/journal.pcbi.1005582 
36. Breakspear, M. Dynamic models of large-scale brain activity. Nature Neuroscience. 2017; 20(3), 340-352. https://doi.org/10.1038/nn.4497

37. Gabay, N. C., \& Robinson, P. A. Cortical geometry as a determinant of brain activity eigenmodes: Neural field analysis. Physical Review E. 2017; 96(3). https://doi.org/10.1103/PhysRevE.96.032413

38. Atasoy, S., Deco, G., Kringelbach, M. L., \& Pearson, J. Harmonic Brain Modes: A Unifying Framework for Linking Space and Time in Brain Dynamics. Neuroscientist. 2018; 24(3), 277-293. https://doi.org/10.1177/1073858417728032

39. Duindam, V., Macchelli A., Stramigioli S. \& Bruyninckx H. Modeling and Control of Complex Physical Systems: The Port-Hamiltonian Approach Berlin: Springer-Verlag; 2009.

40. Lynn, C. W., \& Bassett, D. S. The physics of brain network structure, function, and control. Nature Reviews Physics. 2018; 1(May). https://doi.org/10.1038/s42254-019-0040-8

41. Schellenberger Costa, M., Weigenand, A., Ngo, H. V. V., Marshall, L., Born, J., Martinetz, T., \& Claussen, J. C. A Thalamocortical Neural Mass Model of the EEG during NREM Sleep and Its Response to Auditory Stimulation. PLOS Comp Bio. 2016; 12(9), 1-20. https://doi.org/10.1371/journal.pcbi.1005022

42. Wei, Y., Krishnan, G. P., Komarov, M., \& Bazhenov, M. Differential roles of sleep spindles and sleep slow oscillations in memory consolidation. PLOS Computational Biology. 2018; 14(7), e1006322. https://doi.org/10.1371/journal.pcbi.1006322

43. Wei, Y., Krishnan, G. P., \& Bazhenov, M. Synaptic Mechanisms of Memory Consolidation during Sleep Slow Oscillations. Journal of Neuroscience. 2016; 36(15), 4231-4247. https://doi.org/10.1523/jneurosci.3648-15.2016

44. Black, R. D., \& Rogers, L. L. Sensory Neuromodulation. Frontiers in Systems Neuroscience, 14(March) 2020, 1-20. https://doi.org/10.3389/fnsys.2020.00012

45. Rihm, J. S., Diekelmann, S.,Born, J. \& Rasch, B. Reactivating memories during sleep by odors: odor specificity and associated changes in sleep oscillations. J. Cogn. Neurosci., 2014, 26: 1806-18.

46. Klinzing, J. G., Kugler, S., Soekadar, S. R., Rasch, B., Born, J. \& Diekelmann, S. Odor cueing during slow-wave sleep benefits memory independently of low cholinergic tone. Psychopharmacology (Berl.), 2017: 1-9

47. Pereira, S. I. R., Beijamini, F., Weber, F. D., Vincenzi, R. A., Da Silva, F. a. C. \& Louzada, F. M. Tactile stimulation during sleep alters slow oscillation and spindle densities but not motor skill. Physiol. Behav., 2017, 169: 59-68

48. Marshall, L., Mö lle, M., Hallschmid, M. \& Born, J. Transcranial direct current stimulation during sleep improves declarative memory. J. Neurosci., 2004, 24: 9985-92

49. Marshall, L., Helgadóttir, H., Mö lle, M. \& Born, J. Boosting slow oscillations during sleep potentiates memory. Nature, 2006, 444: 610-13.

50. Marshall, L., Kirov, R., Brade, J., Mö lle, M. \& Born, J. Transcranial electrical currents to probe EEG brain rhythms and memory consolidation during sleep in humans. PLoS ONE, 2011, 6: e16905. 
51. Eggert, T., Dorn, H., Sauter, C., Nitsche, M. A., Bajbouj, M. \& Danker-Hopfe, H. No effects of slow oscillatory transcranial direct current stimulation (tDCS) on sleep-dependent memory consolidation in healthy elderly subjects. Brain Stimulat., 2013, 6: 938-45

52. Sahlem, G. L., Badran, B. W., Halford, J. J. et al. Oscillating square wave transcranial direct current stimulation (tDCS) delivered during slow wave sleep does not improve declarative memory more than sham: a randomized sham controlled crossover study. Brain Stimulat., 2015, 8: 528-34.

53. Westerberg, C. E., Florczak, S. M., Weintraub, S. et al. Memory improvement via slow-oscillatory stimulation during sleep in older adults. Neurobiol. Aging, 2015, 36: 2577-86.

54. . Ezzyat, Y., Wanda, P. A., Levy, D. F., Kadel, A., Aka, A., Pedisich, I. et al. Closed-loop stimulation of temporal cortex rescues functional networks and improves memory. Nature Communications. 2018, https://doi.org/10.1038/s41467-017-02753-0

55. Weigenand, A., Mölle, M., Werner, F., Martinetz, T., \& Marshall, L. Timing matters: open-loop stimulation does not improve overnight consolidation of word pairs in humans. European Journal of Neuroscience. 2016. https://doi.org/10.1111/ejn.13334

56. Ngo, H. V. V., Miedema, A., Faude, I., Martinetz, T., Molle, M., \& Born, J. Driving Sleep Slow Oscillations by Auditory Closed-Loop Stimulation-A Self-Limiting Process. Journal of Neuroscience. 2015; 35(17), 6630-6638. https://doi.org/10.1523/JNEUROSCI.3133-14.2015

57. Woodman, G. F. A brief introduction to use of ERPs in stidues of perception and attention. Attention, Perception, \& Psychophysics. 2010; 72(8), 1-29. https://doi.org/10.3758/APP.72.8.2031.A

58. Deprez, M., Luyck, K., Luyten, L., Tambuyzer, T., Nuttin, B., \& Mc Laughlin, M. An evaluation of the effect of pulse-shape on grey and white matter stimulation in the rat brain. Scientific Reports. 2018; 8(1), 1-10. https://doi.org/10.1038/s41598-017-19023-0

59. O'Reilly, C., \& Nielsen. Automatic sleep spindle detection: benchmarking with fine temporal resolution using open science tools. Frontiers in Human Neuroscience, 2015; 1-19. https://doi.org/10.3389/fnhum.2015.00353

60. Muehlroth, B. E., Sander, M. C., Fandakova, Y., Grandy, T. H., Rasch, B., Shing, Y. L., \& Werkle-Bergner, M. Precise Slow Oscillation-Spindle Coupling Promotes Memory Consolidation in Younger and Older Adults. Scientific Reports. 2019, 9(1), 1-15. https://doi.org/10.1038/s41598-018-36557-z

61. Wavelet python package https://gist/.github.com/patoorio/a960ef16489639fbf20f23ac49ba24f 
A

B

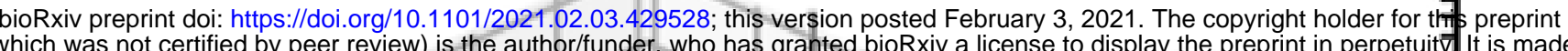

$10^{-3}$
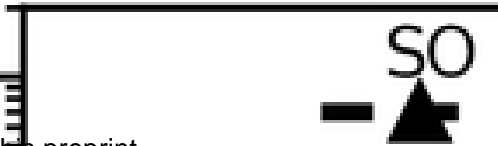

SP

Z

(

Z $0.6 \quad \phi_{n}^{(0)}=2$

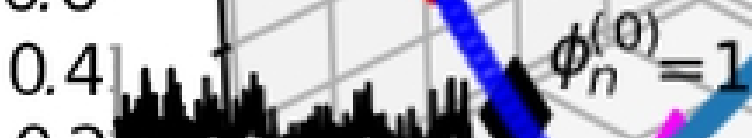

0.2 . nnm

0.0

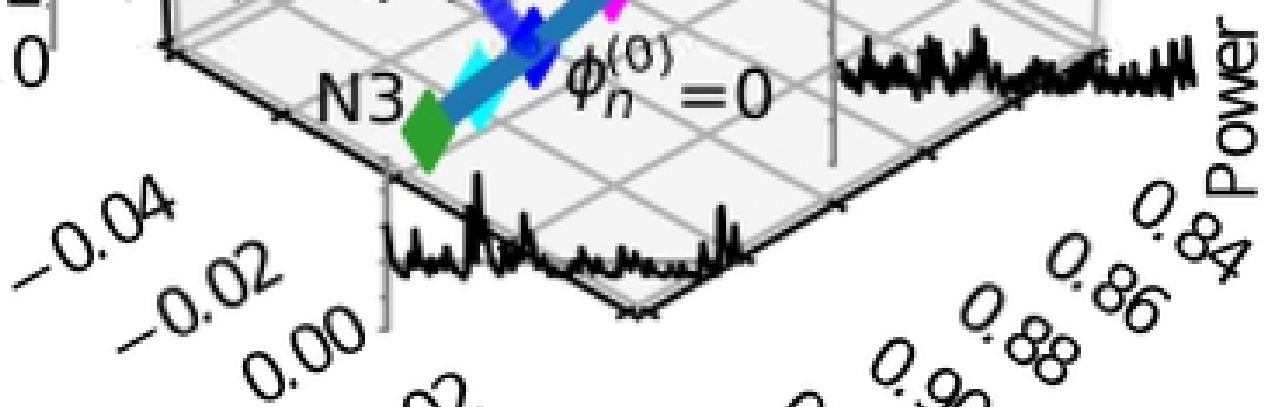
$Y \quad 0.02$ $0.040_{8}^{0}$
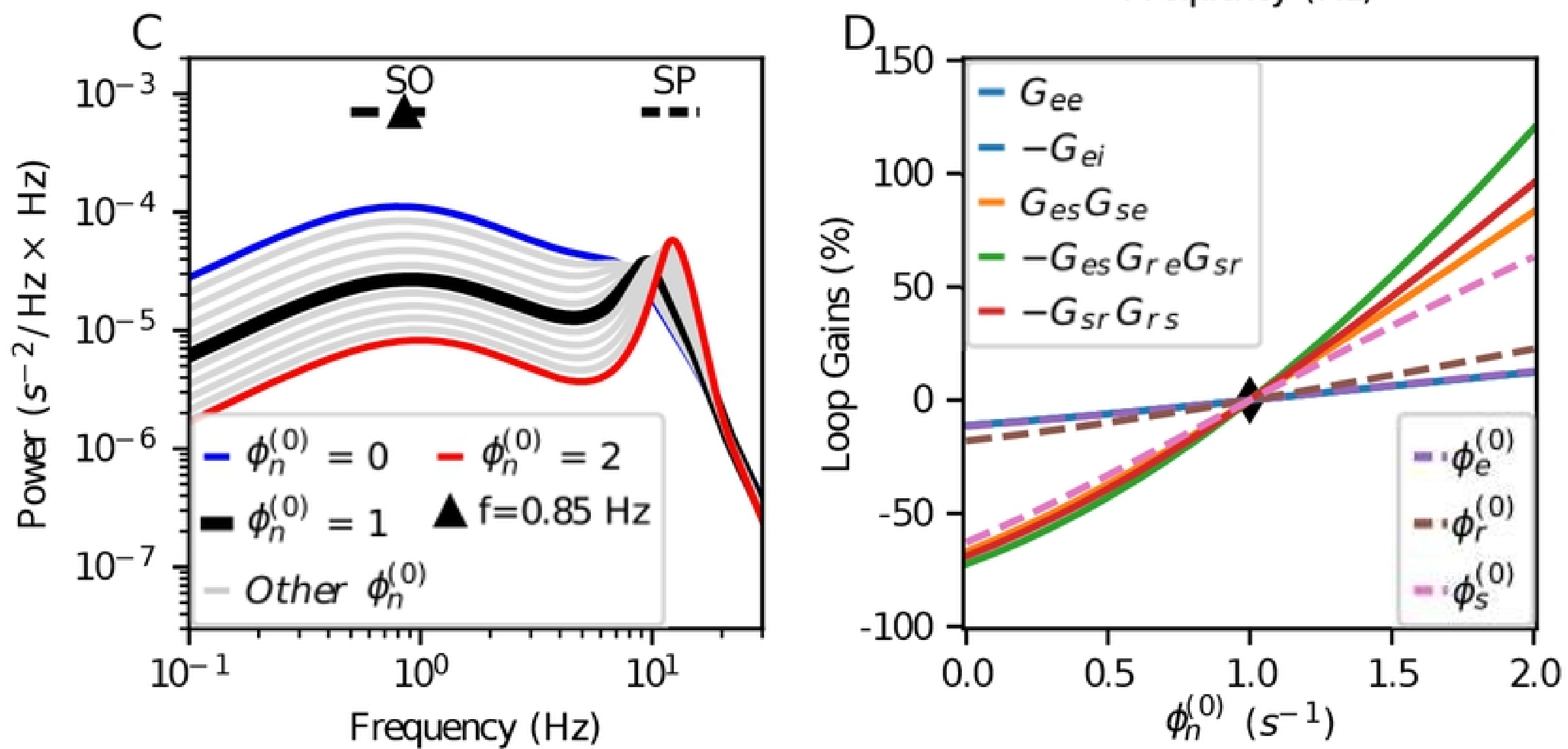

Figure 1 


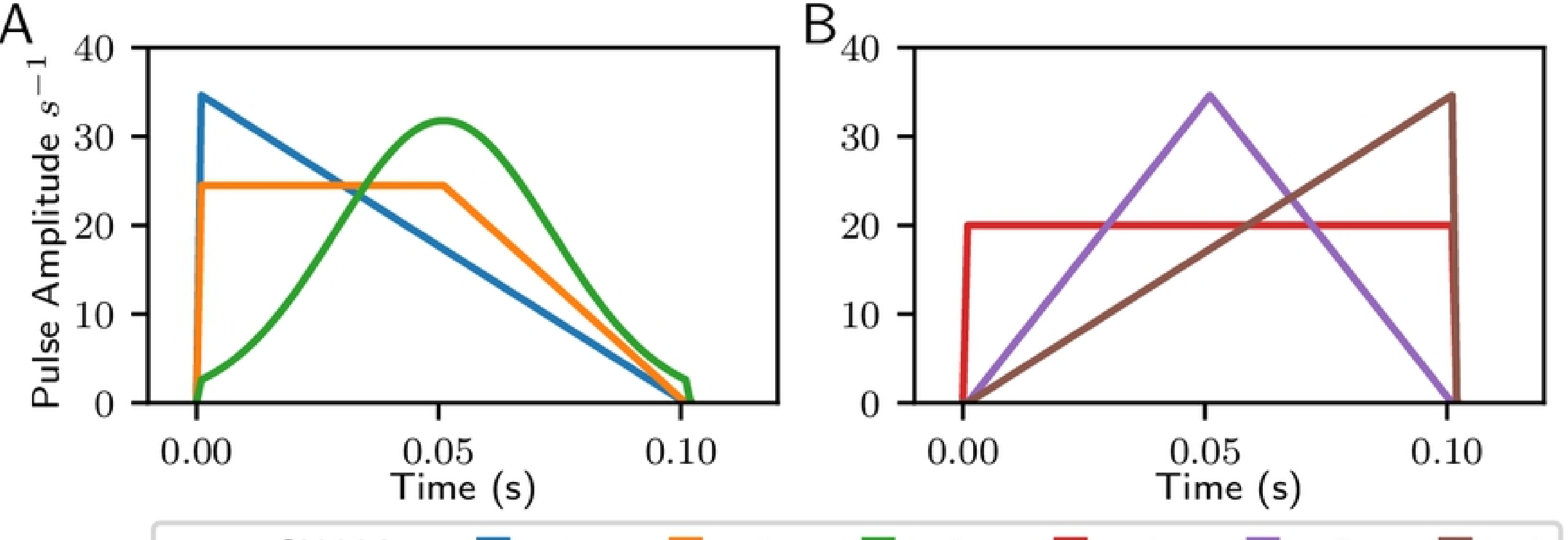

C
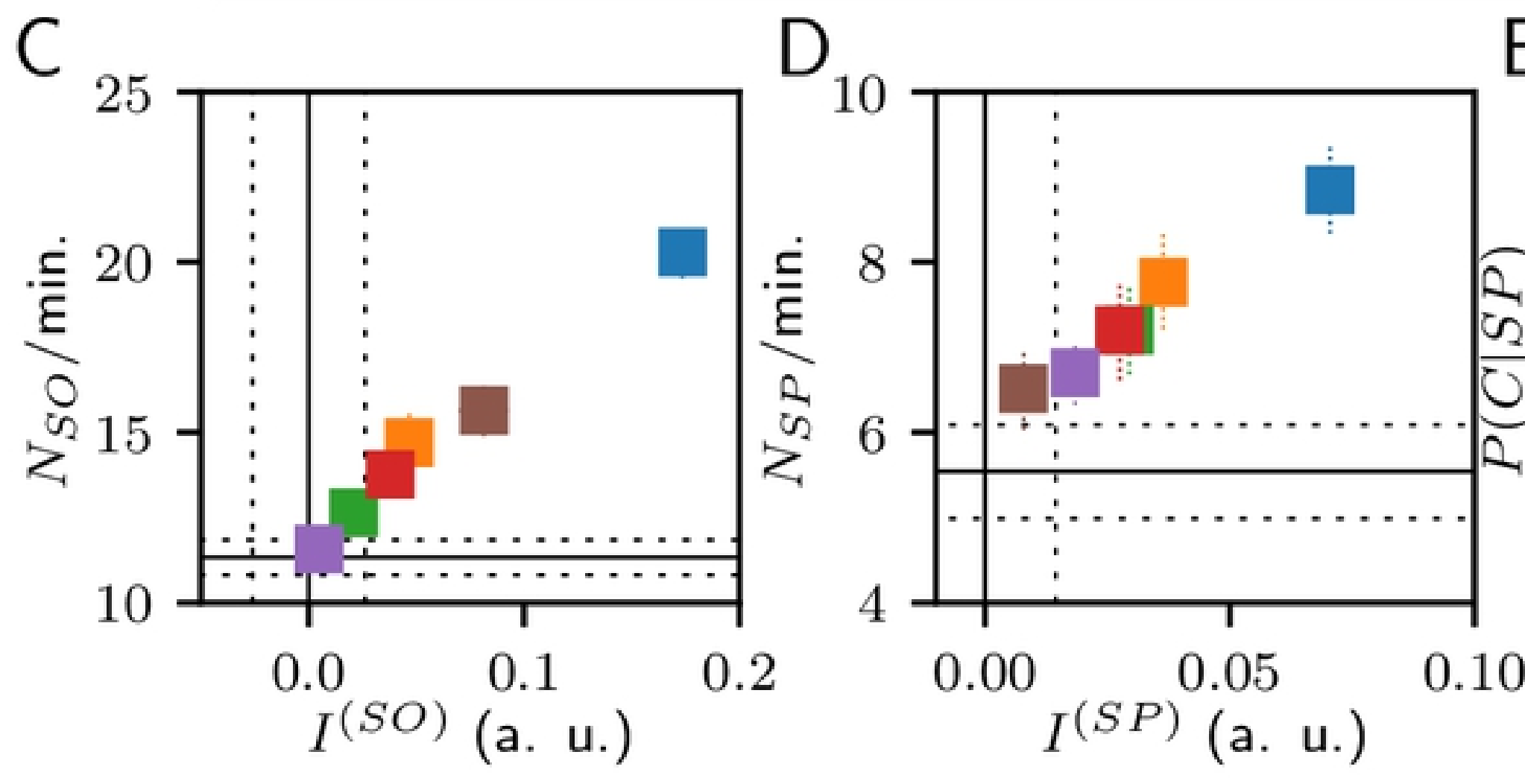

E

Figure 3 

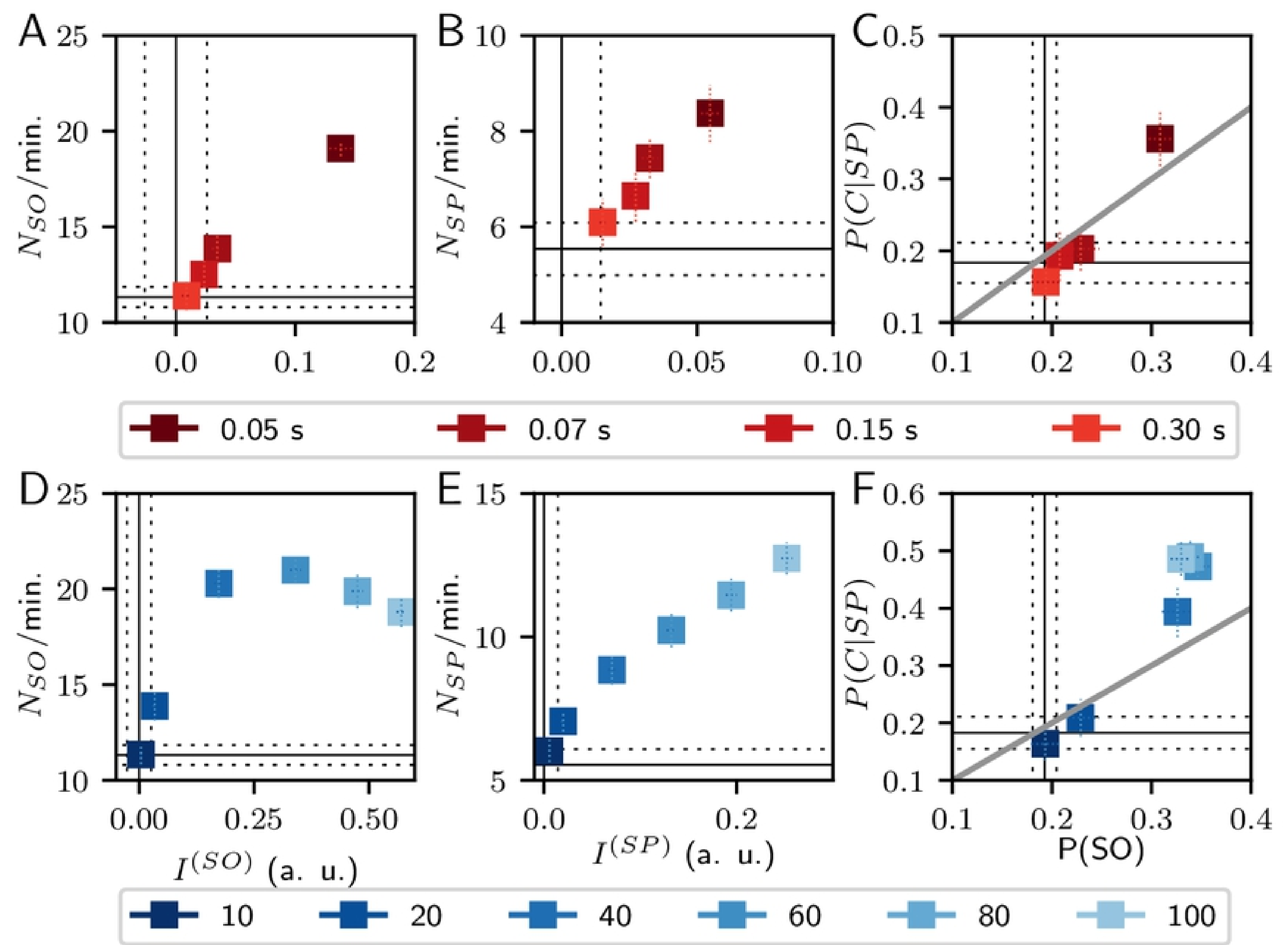

Figure 4 
Envelope detector

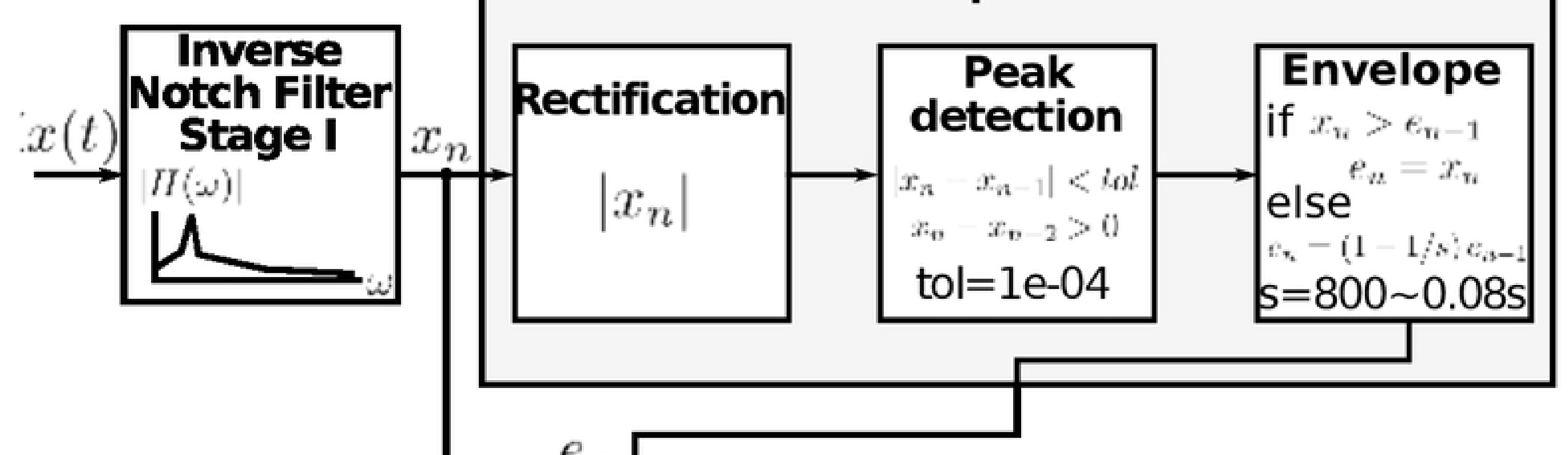
bioRxiv preprint doi: https://doi.org/10.1101/2021. 0.03 .429528 ; this velsibn
(which was not certified by peer review) is the autho /funder, who has granted loRxiv a license to display the preprint in perpetuity. It is made

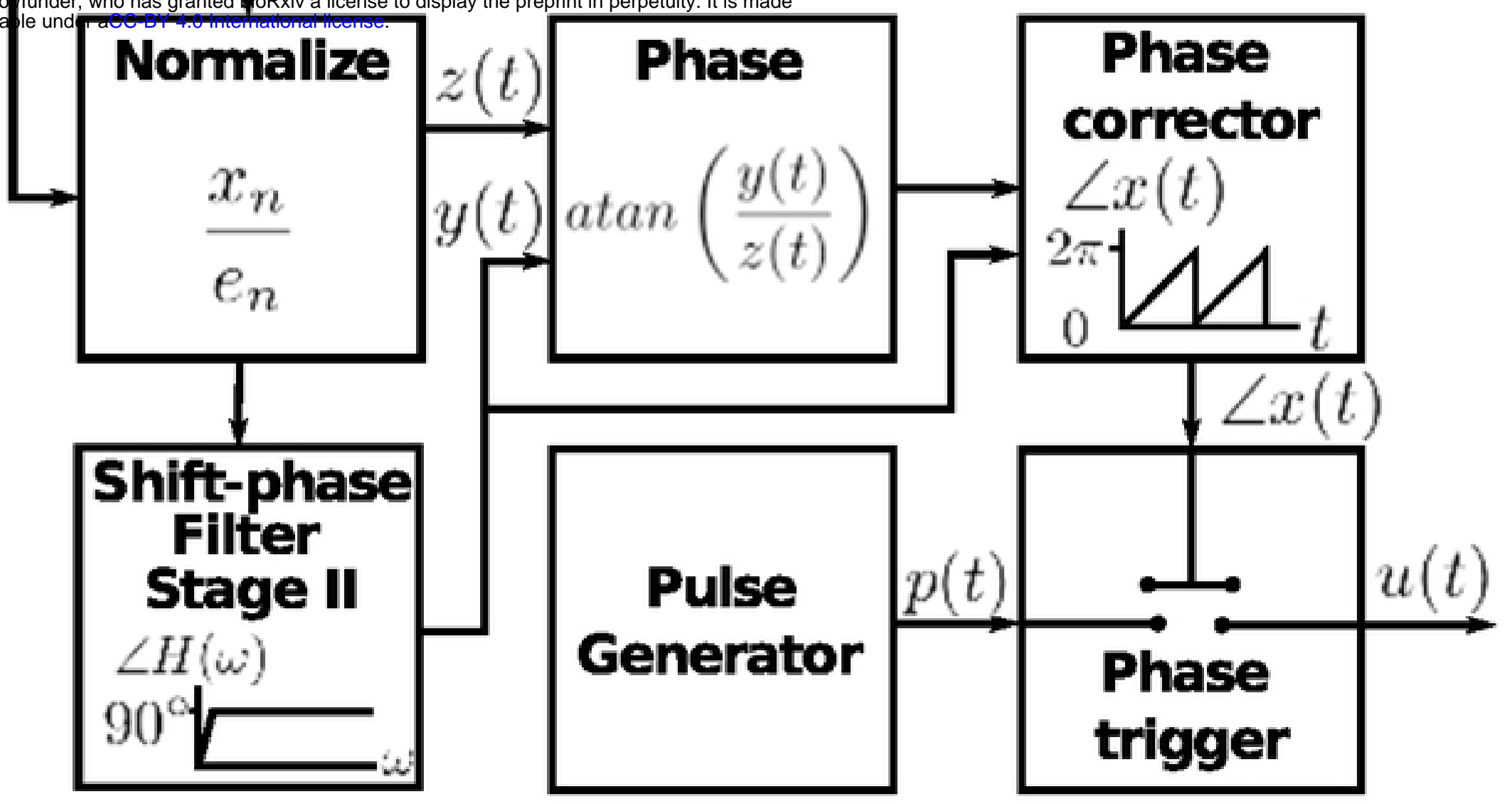

B

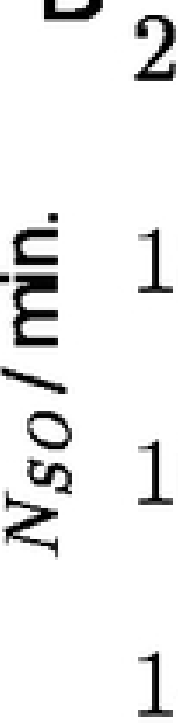

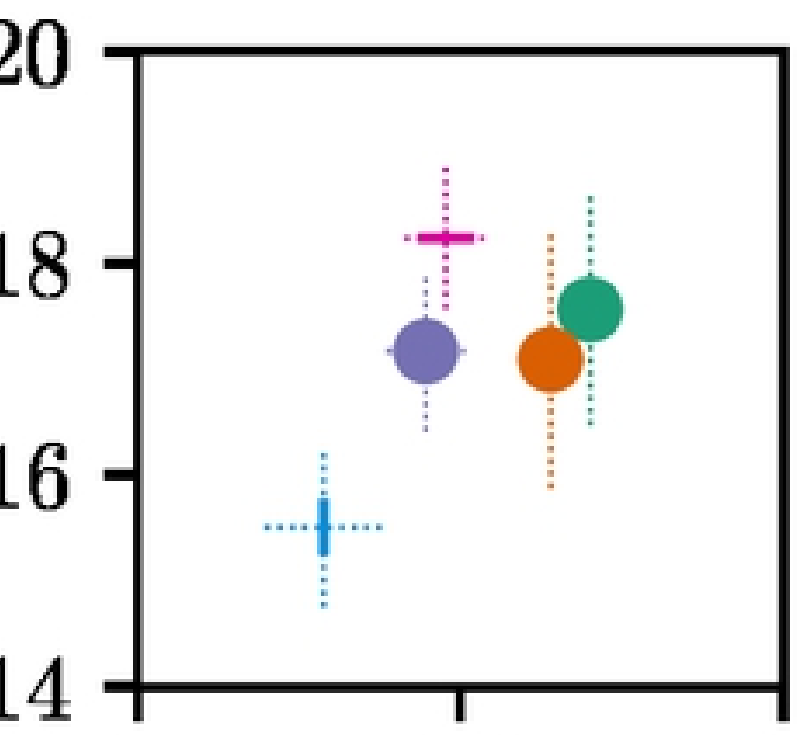

0.1 $I^{(S O)}$ (a. u.)

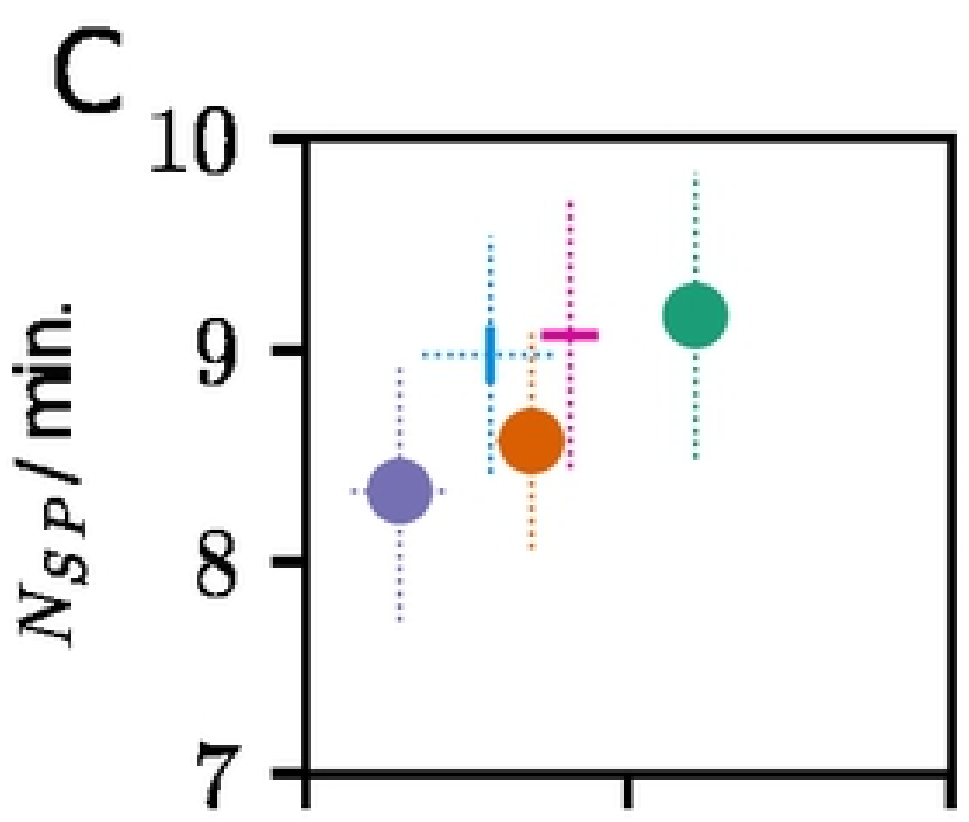

$\begin{array}{lll}0.050 & 0.075 & 0.100\end{array}$ $I^{(S P)}$

(a. u.)

0.3

- STIM-CL 45

- STIM-CL 90

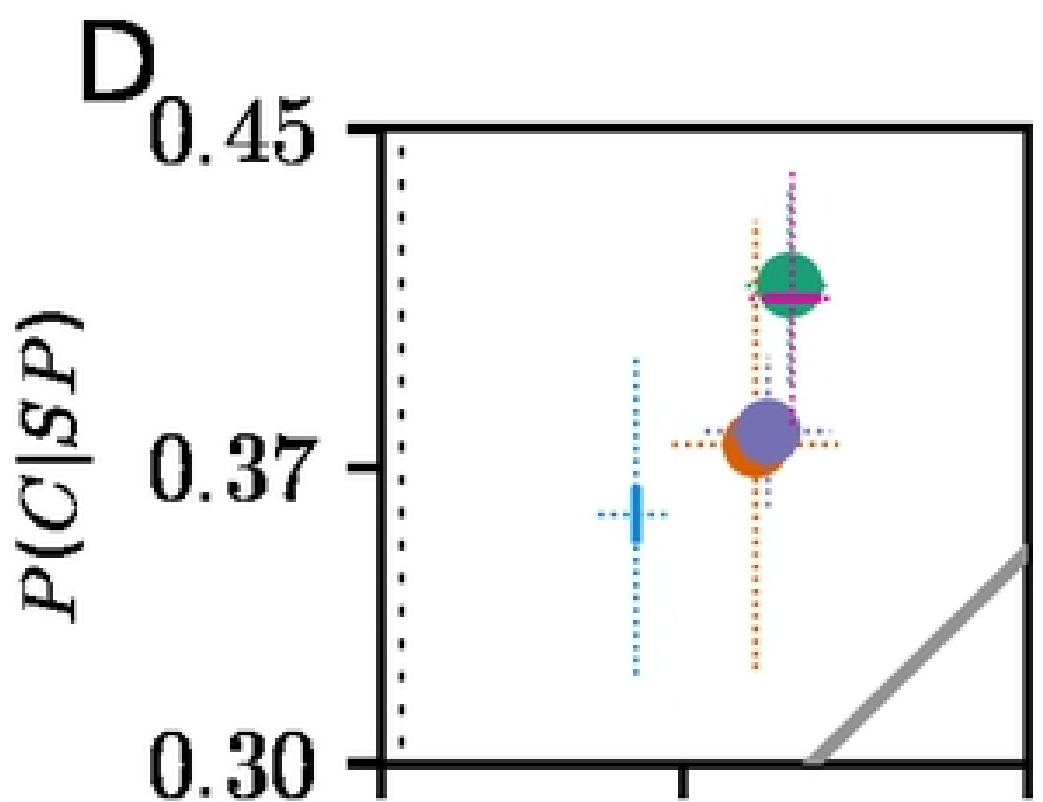

$\begin{array}{ll}0.20 & 0.27\end{array}$ $P(S O)$

Figure 5 
$A \begin{aligned} & \text { bioRxiv preprint doi: } \mathrm{https}: / / \text { doi.org/10.1101/2021.02.03.429528; this version posted February 3, } 222 \text {. The copyright holder for this preprint } \\ & \text { (which was not certified by peer review) is the author/funder, who has granted bioRxiv a license to display the preprint in perpetuity. It is made }\end{aligned}$

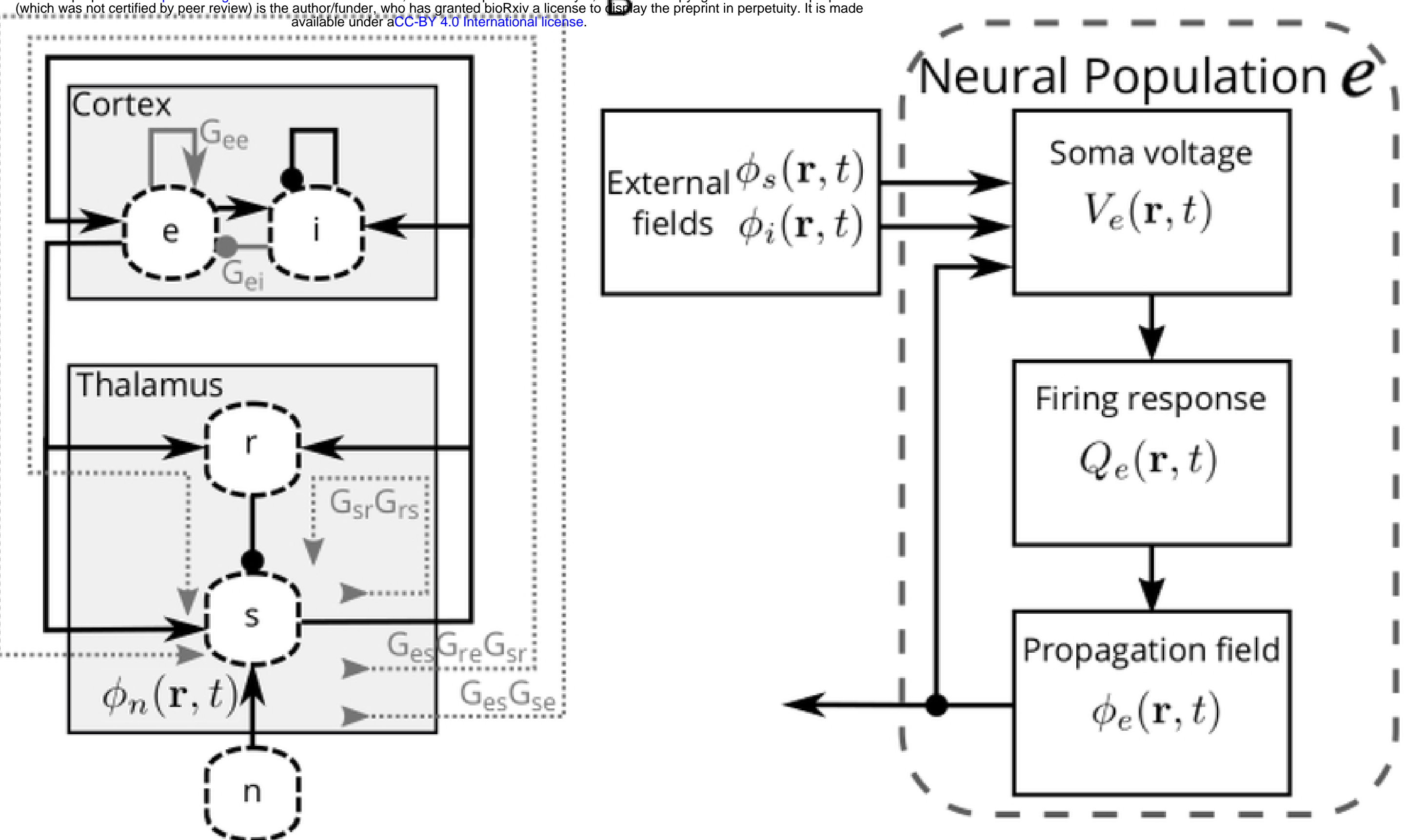

C
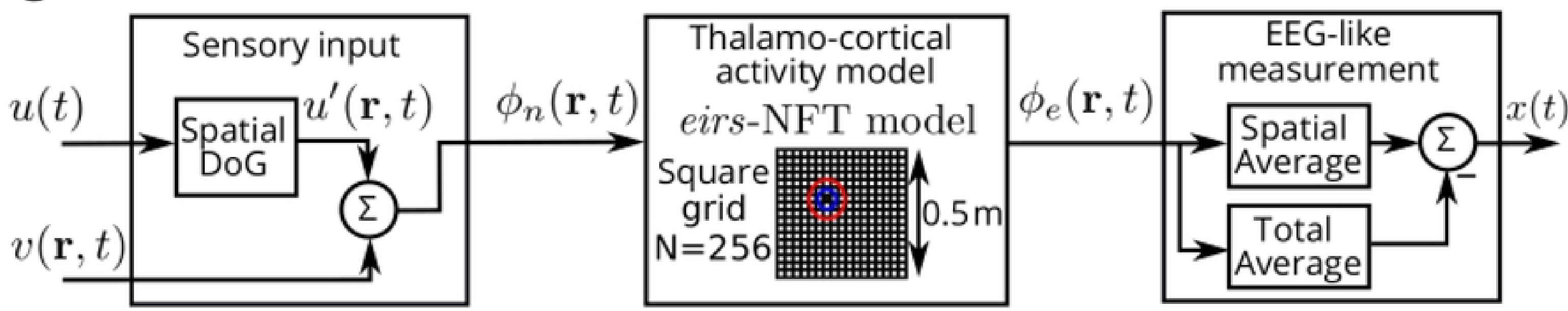

Figure 7 